\title{
Fast and Accurate MoM Analysis of Periodic Arrays of Multilayered Stacked Rectangular Patches With Application to the Design of Reflectarray Antennas
}

\author{
Rafael Florencio, Rafael R. Boix,
}

, and José A. Encinar

\begin{abstract}
The scattering of plane waves by periodic arrays of stacked rectangular patches in multilayered substrates is a problem that has to be solved many times when designing reflectarray antennas made of those patches under the local periodicity assumption. The solution to the periodic multilayered problem has been traditionally carried out by means of the Galerkin's version of the method of moments (MoM) in the spectral domain. This approach involves the computation of double infinite summations, and whereas some of these summations converge very fast, some other converge very slowly. In this paper, the slowly convergent summations are computed by making use of an enhanced mixed potential integral equation (MPIE) formulation of the MoM in the spatial domain. This enhanced formulation is based on the interpolation of the multilayered periodic Green's functions, and on the efficient computation of the four-dimensional (4-D) integrals leading to the MoM matrix entries. Both the novel hybrid spectral-spatial MoM code and the standard spectral domain MoM code have been used for the design of a contoured beam reflectarray antenna. It has been verified that the spectral-spatial MoM code requires CPU times that are typically 30 times smaller than those required by the pure spectral domain MoM code.
\end{abstract}

Index Terms-Green's functions, integral equations, moment methods, multilayered media, periodic structures, reflectarrays.

\section{INTRODUCTION}

$\mathbf{R}$ EFLECTARRAY antennas are an interesting alternative to reflector antennas and phased arrays because of their simple manufacturing process, low weight, improved polarization performance, absence of complex feed networks, etc. [1]. The main disadvantage of reflectarray antennas is their narrow bandwidth, but this disadvantage has been overcome to a large extent by using cells with stacked rectangular patches [2], and by introducing optimization techniques in the design process that adjust the patches dimensions to fulfill the beam requirements at several frequencies [3], [4]. The combined use of cells with stacked patches and wideband optimization techniques has made it possible to design a contoured beam reflectarray for space applications with two independent coverages (European and North American) in orthogonal polarizations at Ku-band [5], a multifed contoured beam reflectarray for central station LMDS applications with three independent beams in alternate polarization at K-band [6] and finally, a transmitreceive reflectarray for DBS applications with dual-frequency dual-polarization capabilities and South American coverage at Ku-band [7].

When designing a frequency optimized reflectarray made of stacked rectangular patches of variable size, it is necessary to accurately tune the sizes of the patches that lead to the appropriate reflection phase at different frequencies for the generation of a given radiation pattern in a prescribed frequency band. In the determination of the sizes of the patches of a certain cell, it is common practice to assume that this cell is surrounded by an infinite periodic array of cells of the same dimensions, which was proposed for the first time by Pozar et al. [8]-[10]. This simplification is known as the local periodicity assumption. The assumption is justified by the fact that it leads to theoretical antenna designs that show a good agreement with measurements [5], [7], [10], [11]. When the local periodicity assumption is used in the design of a frequency-optimized reflectarray made of stacked patches, the numerical analysis of the scattering of a plane wave obliquely incident on a periodic multilayered structure has to be carried out a huge number of times. Therefore, very efficient numerical tools are required for the solution of this scattering problem.

The numerical method traditionally employed for the characterization of reflectarray cells in periodic environments is the method of moments (MoM) in the spectral domain [10]-[12]. Unfortunately, when the MoM in the spectral domain is applied, the matrix entries may be slowly convergent double infinite summations, and the accurate brute force computation of these summations may require a long CPU time. Several approaches have been proposed to accelerate the convergence of these series such as the use of two-dimensional (2-D) fast Fourier transforms [13], [14], the combined use of Kummer's transformation and contour integration in the complex plane [15], or the combined use of Kummer's transformation, Poisson's formula, and Chebyshev polynomial interpolation [16]. The problem with all these approaches is that they are restricted to subsectional basis functions (BFs), and these BFs have been found to be less adequate than entire domain BFs when modeling the current on rectangular patches [17] such as those found in the 
reflectarrays of [2]-[7], [11] (the size of the MoM matrix is substantially smaller when entire domain BFs are used instead of subsectional BF). An alternative to the MoM in the spectral domain for the analysis of multilayered periodic structures is the mixed potential integral equation (MPIE) formulation of the MoM in the spatial domain [18], [19]. In this latter approach, one has to face with the computation of multilayered periodic Green's functions (MPGF) consisting of slowly convergent double infinite summations, and with the computation of fourdimensional (4-D) integrals with singular integrands in case Galerkin's version of the MoM is used. Very efficient strategies have been proposed to accelerate the computation of the double summations leading to the MPGF (see [19] and [20], and references therein). Also, the evaluation of 4-D singular integrals is a problem that has been addressed in detail in [21]-[23] for free-space Green's functions and subsectional BF. However, when multilayered Green's functions and entire domain BFs are involved, to the authors' knowledge, the only available strategy for the accurate determination of these latter 4-D singular integrals is the double exponential formula recently described in [24].

In this paper, the authors focus on the efficient analysis of the periodic arrays of stacked rectangular patches that have been used to design the reflectarray antennas of [2]-[7] under the local periodicity assumption. In particular, a hybrid implementation of the Galerkin's version of the MoM is applied to the analysis of those periodic multilayered structures. On one hand, the MoM matrix entries involving BF of patches located at different metallization levels are efficiently computed in the spectral domain. On the other hand, the MoM matrix entries involving BF of patches located at the same metallization level are computed by means of a MPIE spatial domain formulation. The CPU time required for this spatial domain computation of the MoM matrix entries is substantially reduced by introducing two improvements. The first improvement is that the MPGF with 2-D periodicity for the potentials are judiciously interpolated in the spatial domain [25], [26] in terms of 2D Chebyshev polynomials after extracting the behavior of the MGPF around the source points. The second improvement in the spatial domain computation of the MoM entries has to do with the efficient computation of 4-D singular integrals. For entire domain BFs accounting for edge singularities [17], [27], two of the four integrals leading to the MoM matrix entries are cross-correlations between BFs which can be obtained in closed form [28]. The remaining 2-D integrals are numerically evaluated in an efficient way by extracting the MPGF singularities, and by handling the logarithmic singularities of the crosscorrelations with Ma-Rokhlin-Wandzura (MRW) quadrature rules [29], [30]. The numerical results obtained in the paper show that the novel hybrid spectral-spatial MoM approach typically provides a CPU time reduction of roughly two orders of magnitude with respect to the classical spectral domain approach for an accuracy of two significant figures. Both the spectral-spatial domain software and the spectral domain software have been employed for the design of a contoured beam reflectarray antenna under the local periodicity assumption, and it has been checked that a CPU time reduction between

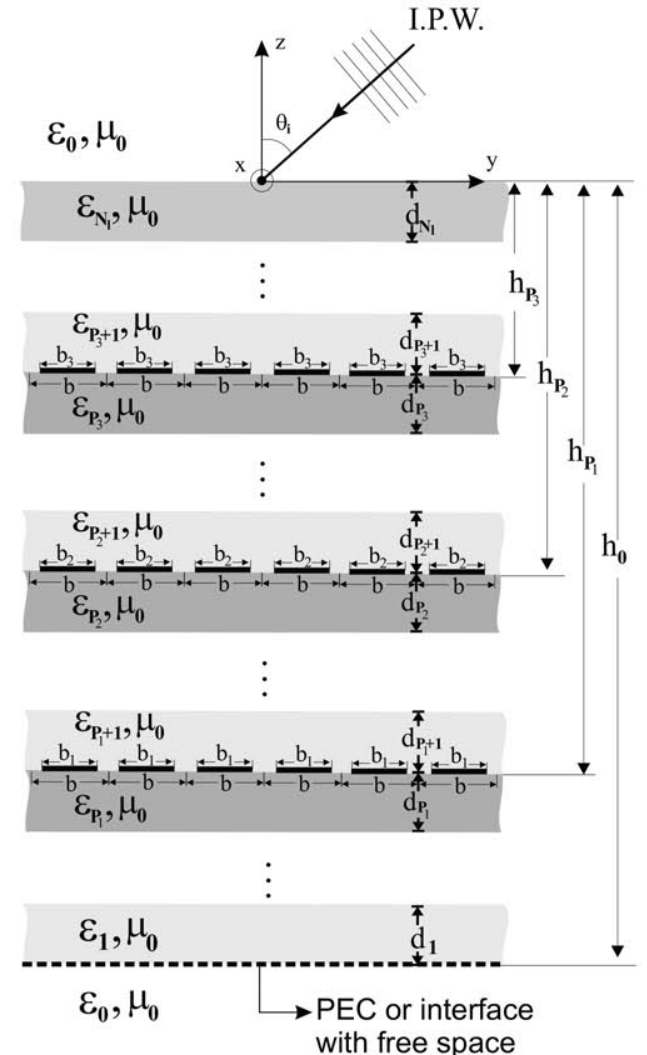

(a)

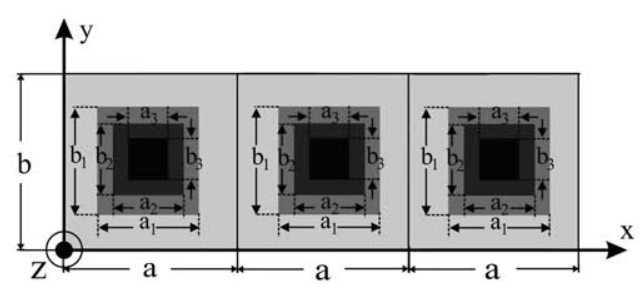

(b)

Fig. 1. (a) Side view and (b) top view of a periodic multilayered structure. The unit cell contains three stacked rectangular patches. The periodic structure is illuminated by a plane wave.

one and two orders of magnitude is achieved for this particular application.

\section{DESCRIPTION OF THE PROBlem}

Fig. 1(a) and (b) shows a periodic multilayered structure containing three stacked rectangular patches in each unit cell. For simplicity, the three patches are assumed to be centered in the periodic unit cell as shown in Fig. 1(b) (however, this restriction is not required in the derivations that follow). The patches are assumed to be PEC with negligible thickness. The multilayered substrate consists of $N_{l}$ lossy dielectric layers of thickness $d_{i}$ and complex permittivity $\varepsilon_{i}=\varepsilon_{0} \varepsilon_{r, i}\left(1-j \tan \delta_{i}\right)$ $\left(i=1, \ldots, N_{l}\right)$, and it is limited either by a ground plane or by free space at the lower end. The periodic structure of Fig. 1(a) and (b) is obliquely illuminated by a linearly polarized plane wave with an arbitrary polarization direction (the incidence 
direction is given by the angular spherical coordinates $\theta_{\mathrm{i}}$ and $\varphi_{\mathrm{i}}$, and the polarization state is characterized by the polarization angle $\gamma$ shown in [28, Fig. 1(c)]). Assuming a time dependence of the type $\mathrm{e}^{j \omega t}$ which will be suppressed throughout, the incident electric field with unit amplitude can be written as shown in [28, eqs. (1)-(3)]. In order to know the electric field scattered by the periodic structure of Fig. 1(a) and (b), we need to determine the current density induced on the three metallized interfaces, $\mathbf{J}_{1}(x, y), \mathbf{J}_{2}(x, y)$, and $\mathbf{J}_{3}(x, y)$, by the incident plane wave. These three current densities can be obtained by solving the system of three coupled electric field integral equations (EFIE) shown as follows:

$$
\begin{aligned}
& \hat{\mathbf{z}} \times\left[\mathbf{E}^{\mathrm{ms}}\left(x, y, z=-h_{P_{i}}\right)\right. \\
& \quad+\sum_{j=1}^{3} \sum_{m=-\infty}^{+\infty} \sum_{n=-\infty}^{+\infty} \int_{a / 2-a_{j} / 2+m a}^{a / 2+a_{j} / 2+m a} \int_{b / 2-b_{j} / 2+n b}^{b / 2+b_{j} / 2+n b} \\
& \overline{\mathbf{G}}^{E}\left(x-x^{\prime}, y-y^{\prime}, z=-h_{P_{i}}, z^{\prime}=-h_{P_{j}}\right) \\
& \left.. \mathbf{J}_{j}\left(x^{\prime}, y^{\prime}\right) d x^{\prime} d y^{\prime}\right]=\mathbf{0} a / 2-a_{i} / 2<x<a / 2+a_{i} / 2 ; \\
& b / 2-b_{i} / 2<y<b / 2+b_{i} / 2 \quad(i=1,2,3)
\end{aligned}
$$

where $\mathbf{E}^{\mathrm{ms}}(x, y, z)$ is the electric field generated in all space by the plane wave impinging on the multilayered substrate in the absence of the patches, and $\overline{\mathbf{G}}^{E}$ is the non-periodic dyadic Green's function of the multilayered substrate [31]. Since $\mathbf{J}_{j}(x, y)(j=1,2,3)$ are Floquet-periodic functions in the $x$ - and $y$-directions, $\mathbf{J}_{j}(x, y)$ can be written as

$$
\mathbf{J}_{j}(x, y)=\sum_{m=-\infty}^{+\infty} \sum_{n=-\infty}^{+\infty} \widetilde{\mathbf{J}}_{j}^{\mathrm{d}}\left(k_{x m}, k_{y n}\right) \mathrm{e}^{\mathrm{j}\left(k_{x m} x+k_{y m} y\right)}
$$

where $k_{x m}=k_{0} \sin \theta_{\mathrm{i}} \cos \varphi_{\mathrm{i}}+2 \pi m / a, k_{y m}=k_{0} \sin \theta_{\mathrm{i}} \sin \varphi_{\mathrm{i}}$ $+2 \pi n / b$, and where

$$
\begin{aligned}
\widetilde{\mathbf{J}}_{j}^{\mathrm{d}}\left(k_{x m}, k_{y n}\right)= & \frac{1}{a b} \\
& \times \int_{a / 2-a_{j} / 2}^{a / 2+a_{j} / 2} \int_{b / 2-b_{j} / 2}^{b / 2+b_{j} / 2} \mathbf{J}_{j}(x, y) \\
& \times \mathrm{e}^{-\mathrm{j}\left(k_{x m} x+k_{y m} y\right)} d x d y .
\end{aligned}
$$

If the MoM is used to solve the system of coupled EFIEs shown in (1), $\mathbf{J}_{j}(x, y)(j=1,2,3)$ have to be expanded in terms of known BFs $\mathbf{J}_{j l}(x, y)\left(l=1, \ldots, N_{b}\right)$ as follows:

$$
\mathbf{J}_{j}(x, y)=\sum_{l=1}^{N_{b}} c_{j l} \mathbf{J}_{j l}(x, y) \quad(j=1,2,3) .
$$

When (4) is introduced in (3), it turns out that the spectral functions of (3) $\widetilde{\mathbf{J}}_{j}^{\mathrm{d}}\left(k_{y m}\right)$ is also expanded in terms of spectral $\mathrm{BF} \widetilde{\mathbf{J}}_{j l}^{\mathrm{d}}\left(k_{y m}\right)(l=1, \ldots, N)$ as follows:

$$
\widetilde{\mathbf{J}}_{j}^{\mathrm{d}}\left(k_{x m}, k_{y n}\right)=\sum_{l=1}^{N_{b}} c_{j l} \widetilde{\mathbf{J}}_{j l}^{\mathrm{d}}\left(k_{x m}, k_{y n}\right) .
$$

When (4) is substituted into (1) and the Galerkin's version of the $\mathrm{MoM}$ is applied, the following system of linear equations for the unknown coefficients $c_{j l}$ is obtained as

$$
\sum_{j=1}^{3} \sum_{l=1}^{N_{b}} \Lambda_{k l}^{i j} c_{j l}=d_{k}^{i} \quad\left(i=1,2,3 ; k=1, \ldots, N_{b}\right) .
$$

By invoking Parseval's identity for 2-D Fourier transforms, the elements $\Lambda_{k l}^{i j}$ of the coefficient matrix of the system of equations (6) can be expressed as double infinite summations of the following type [12]

$$
\begin{aligned}
\Lambda_{k l}^{i j}= & a b \sum_{m=-\infty}^{+\infty} \sum_{m=-\infty}^{+\infty}\left[\left(\widetilde{\mathbf{J}}_{i k}^{\mathrm{d}}\left(k_{x m}, k_{y n}\right)\right)^{*}\right]^{t} \\
& \widetilde{\widetilde{\mathbf{G}}}^{E, \mathrm{c}}\left(k_{x}=k_{x m}, k_{y}=k_{y m}, z=-h_{P_{i}}, z^{\prime}=-h_{P_{j}}\right) \\
& . \widetilde{\mathbf{J}}_{j l}^{\mathrm{d}}\left(k_{x m}, k_{y n}\right) \quad\left(i, j=1,2,3 ; k, l=1, \ldots, N_{b}\right)
\end{aligned}
$$

where $\tilde{\overline{\mathbf{G}}}^{E, \mathrm{c}}\left(k_{x}, k_{y}, z=-h_{P_{i}}, z^{\prime}=-h_{P_{j}}\right)$ is the continuous 2-D Fourier transform of $\overline{\mathbf{G}}^{E}\left(x, y, z=-h_{P_{i}}, z^{\prime}=\right.$ $\left.-h_{P_{j}}\right)$. The components of $\widetilde{\overline{\mathrm{G}}}^{E, \mathrm{c}}\left(k_{x}, k_{y}, z=-h_{P_{i}}, z^{\prime}=\right.$ $-h_{P_{j}}$ ) involved in (7) can be obtained by means of the recurrent algorithm described in [31]. Also, the coefficients $d_{k}^{i}$ of the system of equations (6) can be obtained in the spectral domain as

$$
\begin{aligned}
d_{k}^{i}= & -a b\left[\left(\widetilde{\mathbf{J}}_{i k}^{\mathrm{d}}\left(k_{x 0}, k_{y 0}\right)\right)^{*}\right]^{t} \cdot \mathbf{E}^{\mathrm{ms}}\left(x, y, z=-h_{P_{i}}\right) \\
& \times \mathrm{e}^{-\mathrm{j} k_{0}\left(\sin \theta_{\mathrm{j}} \cos \varphi_{\mathrm{i}} x+\sin \theta_{\mathrm{i}} \sin \varphi_{\mathrm{i}} y\right)} \\
& \left(i=1,2,3 ; k=1, \ldots, N_{b}\right) .
\end{aligned}
$$

Equations (6)-(8) summarize the spectral domain MoM analysis of the scattering problem shown in Fig. 1(a) and (b). It can be analytically shown that the terms involved in the computation of $\Lambda_{k l}^{i j}$ show an exponential convergence of the type $\mathrm{e}^{-\sqrt{k_{x m}^{2}+k_{y n}^{2}}\left|h_{P_{i}}-h_{P_{j}}\right|}$ as $k_{x m}^{2}+k_{y n}^{2} \rightarrow \infty$ when $i \neq j$. In practical cases, this means we just need to add a few hundred terms to compute the series $\Lambda_{k l}^{i j}(i \neq j)$ of (7) with a great degree of accuracy, and the computation of $\Lambda_{k l}^{i j}(i \neq j)$ can be confidently carried out in the spectral domain. However, the series involved in the computation of $\Lambda_{k l}^{i i}(i=1,2,3)$ are slowly convergent, and several million terms may be required for the accurate computation of these series as will be shown in Section V. Therefore, the computation of $\Lambda_{k l}^{i i}(i=1,2,3)$ should not be carried out in the spectral domain. In fact, this computation can be made more efficient if it is carried out in the spatial domain in terms of Green's functions for the vector potential and scalar potential of the multilayered substrate of [19, Fig. 1(a)]. If we invoke the rationale followed to obtain [28, eqs. (16), (33), and (34)], it can be shown that the MoM matrix entries $\Lambda_{k l}^{i i}$ can be rewritten as

$$
\begin{aligned}
\Lambda_{k l}^{i i}= & -j \omega T_{i k l}^{A}-\frac{1}{j \omega} T_{i k l}^{\phi} \\
& \quad\left(i=1,2,3 ; k, l=1, \ldots, N_{b}\right)
\end{aligned}
$$


where $T_{i k l}^{A}$ and $T_{i k l}^{\phi}$ are given by the 2-D integrals

$$
\begin{aligned}
T_{i k l}^{A}= & \int_{-a_{i}}^{a_{i}} \int_{-b_{i}}^{b_{i}} g_{i k l}^{A}(x, y) \\
& \times G_{x x}^{A, \operatorname{pp}}\left(x, y, z=-h_{P_{i}}, z^{\prime}=-h_{P_{i}}\right) d x d y \\
T_{i k l}^{\phi}= & \int_{-a_{i}}^{a_{i}} \int_{-b_{i}}^{b_{i}} g_{i k l}^{\phi}(x, y) \\
& \times G^{\phi, \operatorname{pp}}\left(x, y, z=-h_{P_{i}}, z^{\prime}=-h_{P_{i}}\right) d x d y .
\end{aligned}
$$

The functions $G_{x x}^{A, \mathrm{pp}}$ and $G^{\phi, \mathrm{pp}}$ of (10) and (11) are MPGF with 2-D periodicity which can be obtained in terms of the spectral Green's functions $\widetilde{G}_{x x}^{A, c}$ and $\widetilde{G}^{\phi, c}$ defined in $[28$, eqs. (13)-(15)] by means of the double infinite summations

$$
\begin{aligned}
& G_{x x}^{A, \mathrm{pp}}\left(x-x^{\prime}, y-y^{\prime}, z=-h_{P_{i}}, z^{\prime}=-h_{P_{i}}\right)=\frac{1}{a b} \\
& \quad \times \sum_{m=-\infty}^{+\infty} \sum_{n=-\infty}^{+\infty} \widetilde{G}_{x x}^{A, \mathrm{c}}\left(k_{\rho}=k_{\rho, m n}, z=-h_{P_{i}}, z^{\prime}=-h_{P_{i}}\right) \\
& \quad \times \mathrm{e}^{\mathrm{j}\left[k_{x m}\left(x-x^{\prime}\right)+k_{y n}\left(y-y^{\prime}\right)\right]} \\
& G^{\phi, \mathrm{pp}}\left(x-x^{\prime}, y-y^{\prime}, z=-h_{P_{i}}, z^{\prime}=-h_{P_{i}}\right)=\frac{1}{a b} \\
& \quad \times \sum_{m=-\infty}^{+\infty} \sum_{n=-\infty}^{+\infty} \widetilde{G}^{\phi, \mathrm{c}}\left(k_{\rho}=k_{\rho, m n}, z=-h_{P_{i}}, z^{\prime}=-h_{P_{i}}\right) \\
& \quad \times \mathrm{e}^{\mathrm{j}\left[k_{x m}\left(x-x^{\prime}\right)+k_{y n}\left(y-y^{\prime}\right)\right]}
\end{aligned}
$$

where $k_{\rho, m n}=\sqrt{k_{x n}^{2}+k_{y m}^{2}}$. The functions $g_{i k l}^{A}$ and $g_{i k l}^{\phi}$ are given by

$$
\begin{aligned}
g_{i k l}^{A}(x, y)= & \int_{-\infty}^{+\infty} \int_{-\infty}^{+\infty} \\
& {\left[\left(\mathbf{J}_{i k}(x+u, y+v)\right)^{*}\right]^{t} \cdot \mathbf{J}_{i l}(u, v) d u d v } \\
g_{i k l}^{\phi}(x, y)= & \int_{-\infty}^{+\infty} \int_{-\infty}^{+\infty} \\
& {\left[\nabla \cdot \mathbf{J}_{i k}(x+u, y+v)\right]^{*}\left[\nabla \cdot \mathbf{J}_{i l}(u, v)\right] }
\end{aligned}
$$

i.e., $g_{i k l}^{A}(x, y)$ is a 2-D cross-correlation between $\mathbf{J}_{i k}(x, y)$ and $\mathbf{J}_{i l}(x, y)$, and $g_{i k l}^{\phi}(x, y)$ is a 2-D cross-correlation between $\nabla$. $\mathbf{J}_{i k}(x, y)$ and $\nabla \cdot \mathbf{J}_{i l}(x, y)$. For the particular case of the BF $\mathbf{J}_{i k}(x, y)$ used in this paper, the cross-correlations of (14) and (15) can be obtained in closed form, which introduces important simplifications in the numerical computation of $T_{i k l}^{A}$ and $T_{i k l}^{\phi}$ via (10) and (11) [28].

Once the coefficients $\Lambda_{k l}^{i j}$ are computed [by using (7) as well as (9), (10), and (11)] and the system of equations (6) is solved, the electric field generated in the regions $z>0$ and $z<-h_{0}$ of Fig. 1(a) and (b) by the incident plane wave can be computed in a straightforward way.

\section{EFFicient COMPUTATION OF THE MPGF With SPECIFIC TREATMENT FOR THIN LAYERS}

The double infinite summations of (12) and (13) are slowly convergent, and the brute force accurate determination of $G^{A, p p}$ and $G^{\phi, p p}$ via (12) and (13) may require the evaluation of hundreds of millions of addends. This circumstance has a deleterious effect on the efficient numerical computation of $T_{i k l}^{A}$ and $T_{i k l}^{\phi}$ by means of (10) and (11). One strategy leading to the efficient computation of the series of (12) and (13) is to apply Kummer's decomposition to these series [32] and use the DCIM proposed in [33] for the determination of the asymptotic behavior of the addends in the spectral domain. This strategy has proven to be useful not only in the efficient computation of three-dimensional (3-D) MPGF with 2-D periodicity [20], [34], but also in the efficient computation of 2-D MPGF with one-dimensional (1-D) periodicity [28].

Let $G_{0 i}^{\mathrm{pp}}(x, y)(i=1,2,3)$ represent any of the two spatial domain MPGF $G_{x x}^{A, p p}\left(x, y, z=-h_{P_{i}}, z^{\prime}=-h_{P_{i}}\right)$ and $G^{\phi, \operatorname{pp}}\left(x, y, z=-h_{P_{i}}, z^{\prime}=-h_{P_{i}}\right)$ of (12) and (13), and let $\widetilde{G}_{0 i}^{c}\left(k_{\rho}\right)(i=1,2,3)$ represent any of the two spectral domain Green's functions $\widetilde{G}_{x x}^{A, \mathrm{c}}\left(k_{\rho}, z=-h_{P_{i}}, z^{\prime}=-h_{P_{i}}\right)$ and $\widetilde{G}^{\phi, \mathrm{c}}\left(k_{\rho}, z=-h_{P_{i}}, z^{\prime}=-h_{P_{i}}\right)$. According to [20], [33], and [34], the DCIM can be used to provide a very accurate approximation of $\widetilde{G}_{0 i}^{\mathrm{c}}$ in the interval $5 k_{0} \leq k_{\rho}<\infty$ as follows:

$$
\begin{aligned}
\left.\widetilde{G}_{0 i}^{\mathrm{c}}\left(k_{\rho}\right)\right|_{k_{\rho} \geq 5 k_{0}} & \approx \frac{e_{i 0}}{\sqrt{k_{\rho}^{2}-\left(k_{i}^{\mathrm{eff}}\right)^{2}}}+\frac{1}{u_{0}} \sum_{j=1}^{N_{D}} e_{i j} \mathrm{e}^{-u_{0} f_{i j}} \\
& =\widetilde{G}_{0 i}^{\infty}, \mathrm{c}\left(k_{\rho}\right)+\widetilde{G}_{0 i}^{\mathrm{GPoF}, \mathrm{c}}\left(k_{\rho}\right)
\end{aligned}
$$

where $u_{0}=\sqrt{k_{\rho}^{2}-k_{0}^{2}}$ and $k_{0}=\omega \sqrt{\varepsilon_{0} \mu_{0}}$. In (16), the fitting unknown coefficients $e_{i j}$ and $f_{i j}\left(i=1,2,3 ; j=1, \ldots, N_{D}\right)$ of (16) are obtained by means of the generalized pencil of functions (GPoF) technique [35]. Numerical simulations have shown that the real part of the exponents $f_{i j}$ of (16) is always positive (i.e., $\operatorname{Re}\left(f_{i j}\right)>0-i=1,2,3 ; j=$ $1, \ldots, N_{D}-$ ), which means that the term $\widetilde{G}_{0 i}^{\mathrm{GPoF}, \mathrm{c}}\left(k_{\rho}\right)$ exponentially decays as $k_{\rho} \rightarrow \infty$. The coefficients $e_{i 0}$ and $k_{i}^{\text {eff }}$ of $\widetilde{G}_{0 i}^{\infty, c}\left(k_{\rho}\right)$ are chosen in this paper as follows:

$$
\begin{aligned}
e_{i 0}^{A} & =\frac{\mu_{0}}{2} \\
e_{i 0}^{\phi} & =\frac{1}{\varepsilon_{0}\left(\varepsilon_{r, P_{i}}+\varepsilon_{r, P_{i}+1}\right)} \\
k_{i}^{\mathrm{eff}, A} & =k_{0} \sqrt{\frac{\varepsilon_{r, P_{i}}+\varepsilon_{r, P_{i}+1}}{2}} \\
k_{i}^{\mathrm{eff}, \phi} & =k_{0} \sqrt{\frac{2 \varepsilon_{r, P_{i}} \varepsilon_{r, P_{i}+1}}{\varepsilon_{r, P_{i}}+\varepsilon_{r, P_{i}+1}}}
\end{aligned}
$$

which is a choice different from those proposed in [28] and [33]. As pointed out in [25], the choice of $e_{i 0}$ and $k_{i}^{\text {eff }}$ carried out in (17)-(20) makes it possible that the first two terms of the asymptotic expansion of $\widetilde{G}_{0 i}^{\infty, c}\left(k_{\rho}\right)$ as $k_{\rho} \rightarrow \infty$ match the same dominant terms of the asymptotic expansion of $\widetilde{G}_{0 i}^{c}\left(k_{\rho}\right)$. As a consequence of this, $\widetilde{G}_{0 i}^{\mathrm{c}}\left(k_{\rho}\right)-\widetilde{G}_{0 i}^{\infty}, \mathrm{c}\left(k_{\rho}\right)$ decays at a rate $k_{\rho}^{-5}$ as $k_{\rho} \rightarrow \infty$ [25]. It has been found that this decaying behavior is very well reproduced by the decaying exponential functions of $\widetilde{G}_{0 i}^{\mathrm{GPoF}, \mathrm{c}}\left(k_{\rho}\right)$. In fact, we have found that the choice of $e_{i 0}$ and $k_{i}^{\text {eff }}$ given by (17)-(20) leads to both increased accuracy and stability in the GPoF approximation of $\widetilde{G}_{0 i}^{c}\left(k_{\rho}\right)$ with respect to other published choices [28], [33]. 
Numerical simulations have shown that the approximation obtained for $\widetilde{G}_{0}^{\mathrm{c}}\left(k_{\rho}\right)$ when (17)-(20) are substituted in (16) provides an accuracy of at least seven significant figures in most of the interval $5 k_{0} \leq k_{\rho}<\infty$. Bearing in mind this fact, we can apply Kummer's transformation [32] to (12) and (13), while using $\widetilde{G}_{0 i}^{\infty, \mathrm{c}}\left(k_{\rho}\right)+\widetilde{G}_{0 i}^{\mathrm{GP} \circ \mathrm{F}, \mathrm{c}}\left(k_{\rho}\right)$ as asymptotic behavior of $\widetilde{G}_{0 i}^{c}\left(k_{\rho}\right)$ when $k_{\rho} \rightarrow \infty$. After application of Kummer's transformation, a version of [28, eq. (24)] is obtained in which the three single summations are substituted by three double summations. The double summation involving $\widetilde{G}_{0 i}^{\mathrm{c}}\left(k_{\rho}=k_{\rho, m n}\right)-$ $\widetilde{G}_{0 i}^{\infty, c}\left(k_{\rho}=k_{\rho, m n}\right)-\widetilde{G}_{0 i}^{\mathrm{GPoF}, \mathrm{c}}\left(k_{\rho}=k_{\rho, m n}\right)$ requires the computation of less than 100 terms provided $a<\lambda_{0}$ and $b<\lambda_{0}$ $\left(\lambda_{0}=\frac{2 \pi}{k_{0}}\right)$, which is a situation encountered in many practical applications [20] (e.g., in the design of reflectarray antennas to avoid the appearance of grating lobes). The other two asymptotic double summations involving $\widetilde{G}_{0 i}^{\infty}{ }^{\infty}\left(k_{\rho}=k_{\rho, m n}\right)$ and $\widetilde{G}_{0 i}^{\mathrm{GPoF}, \mathrm{c}}\left(k_{\rho}=k_{\rho, m n}\right)$ can be expressed in the spatial domain by means of Poisson's formula, and after application of Poisson's formula, they can be efficiently computed by means of Ewald's method for periodic Green's functions with 2-D periodicity in homogeneous media as shown in [32], [34], and [36].

Bearing in mind the ideas presented in [25], further CPU time reductions can be achieved in the numerical computation of the 2-D integrals of (10) and (11) if the MPGF $G_{0 i}^{\mathrm{pp}}(x, y)$ are interpolated in terms of the two variables $x$ and $y$ in one of the unit cells of the periodic structure of Fig. 1(a) and (b). However, according to [25], the Green's functions $G_{0 i}^{\mathrm{pp}}(x, y)$ should be regularized before proceeding with the interpolation, i.e., the singularities of $G_{0 i}^{\mathrm{pp}}(x, y)$ at the source points as well as the singularities of the derivatives of $G_{0 i}^{\mathrm{PP}}(x, y)$ should be extracted in closed form prior to the interpolation. Let us focus on the singularity of $G_{0 i}^{\mathrm{pp}}(x, y)$ at the source point located at $(x=$ $0, y=0)$ [the source points of $G_{0 i}^{\mathrm{pp}}(x, y)$ are located at $(x=$ $m a, y=n b) ; m, n=\ldots,-2,-1,0,1,2, \ldots]$. The behavior of $G_{0 i}^{\mathrm{pp}}(x, y)$ around $(x=0, y=0)$ is the same as that of the nonperiodic multilayered Green's function around $(x=0, y=0)$, and according to the theory of Fourier transforms, this latter behavior is closely related to the behavior of $\widetilde{G}_{0 i}^{\mathrm{c}}\left(k_{\rho}\right)$ as $k_{\rho} \rightarrow$ $\infty$. If we use the approximation (16) for the computation of $\widetilde{G}_{0 i}^{c}\left(k_{\rho}\right)$ when $k_{\rho} \geq 5 k_{0}$, the behavior of $\widetilde{G}_{0 i}^{c}\left(k_{\rho}\right)$ as $k_{\rho} \rightarrow \infty$ is dominated by the term $e_{i 0} / \sqrt{k_{\rho}^{2}-\left(k_{i}^{\text {eff }}\right)^{2}}$ [remind numerical simulations have shown that $\widetilde{G}_{0 i}^{\mathrm{GPoF}, \mathrm{c}}\left(k_{\rho}\right)$ exponentially decays as $k_{\rho} \rightarrow \infty$ ]. In particular, if we retain the first two dominant terms in the asymptotic expansion of $e_{i 0} / \sqrt{k_{\rho}^{2}-\left(k_{i}^{\text {eff }}\right)^{2}}$ for large $k_{\rho}$, we can write

$$
\left.\widetilde{G}_{0 i}^{\mathrm{c}}\left(k_{\rho}\right)\right|_{k_{\rho} \rightarrow \infty} \approx e_{i 0}\left[\frac{1}{k_{\rho}}+\frac{\left(k_{i}^{\mathrm{eff}}\right)^{2}}{2 k_{\rho}^{3}}\right]=\widetilde{G}_{0 i}^{\mathrm{as} 1, \mathrm{c}}\left(k_{\rho}\right)
$$

Since the behavior of the Fourier transform of a function for large values of the spectral variable is related to the behavior of the function for low values of the spatial variable, both the behavior of the nonperiodic multilayered Green's function and the behavior of $G_{0 i}^{\mathrm{pP}}(x, y)$ when $\rho=\sqrt{x^{2}+y^{2}} \rightarrow 0$ should be dominated by the inverse Hankel transform [see [19, eq. (34) and (35)], when $n=0$ ] of (21), which can be computed by using [37, eq. (6.621.4)] in the Abel sense as

$$
\left.G_{0 i}^{\mathrm{pp}}(x, y)\right|_{\rho \rightarrow 0} \approx \frac{e_{i 0}}{2 \pi}\left[\frac{1}{\rho}-\frac{\left(k_{i}^{\mathrm{eff}}\right)^{2} \rho}{2}\right]=G_{0 i}^{\mathrm{nf} 1, \mathrm{pp}}(x, y) .
$$

Let us now introduce regularization functions that contain the behavior of the nonperiodic multilayered Green's functions around the source point. According to (22), these regularization functions can be defined as

$$
f_{i}^{\mathrm{reg}}(x, y)=G_{0 i}^{\mathrm{nf} 1, \mathrm{pp}}(x, y)=\frac{e_{i 0}}{2 \pi}\left[\frac{1}{\rho}-\frac{\left(k_{i}^{\mathrm{eff}}\right)^{2} \rho}{2}\right] .
$$

Bearing in mind the definition of (23), the regularized MPGF $G_{0 i}^{\mathrm{pp}, \mathrm{reg}}(x, y)$ in the unit cell $\{0 \leq x \leq a ; 0 \leq y \leq b\}$ can be defined as

$$
\begin{aligned}
G_{0 i}^{\mathrm{pp}, \mathrm{reg}}(x, y)= & G_{0 i}^{\mathrm{pp}}(x, y)-f_{i}^{\mathrm{reg}}(x, y) \\
& -\mathrm{e}^{\mathrm{j} k_{x 0} a} f_{i}^{\mathrm{reg}}(x-a, y)-\mathrm{e}^{\mathrm{j} k_{y 0} b} f_{i}^{\mathrm{reg}}(x, y-b) \\
& -\mathrm{e}^{\mathrm{j}\left(k_{x 0} a+k_{y 0} b\right)} f_{i}^{\mathrm{reg}}(x-a, y-b) \\
0 \leq & x \leq a ; 0 \leq y \leq b
\end{aligned}
$$

where the regularization function has been extracted at the four source points placed at the corners of the unit cell. The regularized MPGF of (23) and (24) are basically shown in [25, eq. (25)], except for the fact that Valerio et al. only extract the regularization function at the source point $(x=0, y=0)$ since they work with the unit cell $\{-a / 2 \leq x \leq a / 2 ;-b / 2 \leq y \leq$ $b / 2\}$.

Once the periodic Green's functions have been regularized by extracting the singularities as well as the singularities of the first derivatives [25], the regularized Green's functions $G_{0 i}^{\mathrm{pp}, \text { reg }}(x, y)$ are amenable to 2-D interpolation in terms of $x$ and $y$. While 2-D B-splines were used in [25] for the interpolation of the regularized MPGF with 2-D periodicity, in this paper, we use 2-D Chebyshev polynomials, which are simpler to use (just as we used 1-D Chebyshev polynomials in [28] for the interpolation of regularized MPGF with 1-D periodicity). The resulting interpolating expressions for $G_{0 i}^{\mathrm{pp} \text {, reg }}(x, y)$ in the unit cell $\{0 \leq x \leq a ; 0 \leq y \leq b\}$ are

$$
G_{0 i}^{\mathrm{pp}, \mathrm{reg}}(x, y) \approx \sum_{m=0}^{N_{c}} \sum_{n=0}^{N_{c}} h_{m n}^{i} T_{m}\left(\frac{2 x}{a}-1\right) T_{n}\left(\frac{2 y}{b}-1\right)
$$

where the coefficients $h_{m n}^{i}$ of (25) are computed as

$$
h_{m n}^{i}=\frac{\sum_{k=1}^{N_{c}+1} \sum_{l=1}^{N_{c}+1} w_{k l}^{i} T_{m}\left(s_{k}\right) T_{n}\left(s_{l}\right)}{\left[\sum_{k=1}^{N_{c}+1}\left(T_{m}\left(s_{k}\right)\right)^{2}\right]\left[\sum_{l=1}^{N_{c}+1}\left(T_{n}\left(s_{l}\right)\right)^{2}\right]}
$$

and where $s_{k}=-\cos \left(\frac{2 k-1}{2\left(N_{c}+1\right)} \pi\right)\left(k=1, \ldots, N_{c}+1\right)$ and $w_{k l}^{i}=G_{0 i}^{\mathrm{pp},{ }^{\mathrm{reg}}}\left(x=0.5 a\left(s_{k}+1\right), y=0.5 b\left(s_{l}+1\right)\right)(i=1,2$, $\left.3 ; k, l=1, \ldots, N_{c}+1\right)$. Once an interpolating expression is available for $G_{0 i}^{\mathrm{pp}}(x, y)$ in the unit cell $\{0 \leq x \leq a ; 0 \leq y \leq$ $b\}$ by means of (24) and (25), the computation of $G_{0 i}^{\mathrm{pp}}(x, y)$ 
outside the unit cell can be carried out by invoking the Floquetperiodicity property of $G_{0 i}^{\mathrm{pp}}$ given by

$$
\begin{array}{r}
G_{0 i}^{\mathrm{pp}}(x+m a, y+n b)=\mathrm{e}^{\mathrm{j}\left(k_{x 0} m a+k_{y 0} n b\right)} G_{0 i}^{\mathrm{pp}}(x, y) \\
(m, n=\ldots,-1,0,1, \ldots) .
\end{array}
$$

A common situation encountered in multilayered reflectarray antennas made of stacked patches is that the layers next to the metallizations [located at $z=-h_{P_{i}}$ in Fig. 1(a)] are very thin by comparison with the rest of the layers. This circumstance has a deleterious effect on the interpolation of the Green's function for the scalar potential $G^{\phi, \mathrm{pP}}\left(x-x^{\prime}, y-\right.$ $\left.y^{\prime}, z=-h_{P_{i}}, z^{\prime}=-h_{P_{i}}\right)$. This is because even though the regularized MPGF of (23) and (24) are not singular at the source points, it is nearly singular at these points owing to the presence of very close quasidynamic images [38] through the thin layers in the $z$-direction. This problem could be avoided if the regularization functions of (23) were substituted by regularization functions including the closest quasidynamic images. Although this can be done in closed form as shown in [38], we have noticed that better results are obtained in the interpolation process when the closest images are accounted for in an approximate form by invoking the DCIM. In particular, if we use the approximation (16) for the computation of $\widetilde{G}_{0 i}^{\mathrm{c}}\left(k_{\rho}\right)$ when $k_{\rho} \geq 5 k_{0}$, the asymptotic behavior of $\widetilde{G}_{0 i}^{\mathrm{c}}\left(k_{\rho}\right)$ for large $k_{\rho}$ can be approximately written as

$$
\begin{aligned}
\left.\widetilde{G}_{0 i}^{\mathrm{c}}\left(k_{\rho}\right)\right|_{k_{\rho} \rightarrow \infty} \approx & e_{i 0}\left[\frac{1}{k_{\rho}}+\frac{\left(k_{i}^{\mathrm{eff}}\right)^{2}}{2 k_{\rho}^{3}}+\frac{3\left(k_{i}^{\mathrm{eff}}\right)^{4}}{8 k_{\rho}^{5}}\right. \\
& \left.+\frac{15\left(k_{i}^{\mathrm{eff}}\right)^{6}}{48 k_{\rho}^{7}}\right]+\frac{1}{u_{0}} \sum_{j=1}^{N_{D}} e_{i j} \\
& \times \mathrm{e}^{-u_{0} f_{i j}}=\widetilde{G}_{0 i}^{\mathrm{as} 2, \mathrm{c}}\left(k_{\rho}\right)
\end{aligned}
$$

where four terms have been retained in the asymptotic expansion of $e_{i 0} / \sqrt{k_{\rho}^{2}-\left(k_{i}^{\text {eff }}\right)^{2}}$ instead of retaining two terms as in (21). While the asymptotic behavior of $\widetilde{G}_{0 i}^{\mathrm{c}}\left(k_{\rho}\right)$ for large $k_{\rho}$ of (21) only provides information about the two media next to the source located at $z=-h_{P_{i}}$ as if these two media were semi-infinite, the asymptotic behavior of (28) also provides information about the quasidynamic images of the source through the rest of the layers of the multilayered substrate in an approximate way. Therefore, if we follow once again the rationale used to obtain (22) and (23), the inverse Hankel transform of (28) should provide a more accurate approximation of $G_{0 i}^{\mathrm{pp}}(x, y)$ when $\rho=\sqrt{x^{2}+y^{2}} \rightarrow 0$, especially when the layers next to the metallizations located at $z=-h_{P_{i}}$ are very thin. This inverse Hankel transform can then be used to define a new regularization function $f_{i}^{\text {reg }}(x, y)$ to be used in (24) which is given by

$$
\begin{aligned}
f_{i}^{\mathrm{reg}}(x, y)= & G_{0 i}^{\mathrm{nf} 2, \mathrm{pp}}(x, y)=\frac{e_{i 0}}{2 \pi}\left[\frac{1}{\rho}-\frac{\left(k_{i}^{\mathrm{eff}}\right)^{2} \rho}{2}\right. \\
& \left.+\frac{\left(k_{i}^{\mathrm{eff}}\right)^{4} \rho^{3}}{24}-\frac{\left(k_{i}^{\mathrm{eff}}\right)^{6} \rho^{5}}{720}\right] \\
& +\sum_{j=1}^{N_{D}} \frac{e_{i j}}{2 \pi} \frac{\mathrm{e}^{-j k_{0} \sqrt{\rho^{2}+\left(f_{i j}\right)^{2}}}}{\sqrt{\rho^{2}+\left(f_{i j}\right)^{2}}} .
\end{aligned}
$$

Note that in this paper, the DCIM is used in two ways. On one hand, the DCIM is used in (16) for the approximation of the spectral domain multilayered Green's function, and is subsequently used in Kummer's transformation for the efficient computation of the MPGF. On the other hand, the DCIM is used in (29) to approximately account for the quasidynamic images in the regularization of the MPGF in the cases where thin layers are located next to the metallizations of Fig. 1(a).

\section{BASIS FUNCTIONS AND COMPUTATION OF GALERKIN's MATRIX ENTRIES}

In this paper, the BFs used to model the current density on the rectangular patches of Fig. 1(a) and (b) are Chebyshev polynomials weighted by an edge condition function. These BFs have the advantage that ensure a uniform and fast convergence of the MoM with respect to the number of BFs as reported in [27]. The BFs are as follows:

$$
\begin{aligned}
& \mathbf{J}_{j l}(x, y)=j_{x j}^{r s}(x, y) \hat{\mathbf{x}}=-\frac{\mathrm{j} k_{0}}{2 r b_{j}} U_{r-1}\left(\frac{2(x-a / 2)}{a_{j}}\right) \\
& \times \sqrt{1-\left(\frac{2(x-a / 2)}{a_{j}}\right)^{2}} \frac{T_{s-1}\left(\frac{2(y-b / 2)}{b_{j}}\right)}{\sqrt{1-\left(\frac{2(y-b / 2)}{b_{j}}\right)^{2}}} \hat{\mathbf{x}} \\
& \left(j=1,2,3 ; r=1, \ldots, M_{x}-1\right. \text {; } \\
& \left.s=1, \ldots, M_{y} ; l=1, \ldots,\left(M_{x}-1\right) M_{y}\right) \\
& \mathbf{J}_{j,\left(M_{x}-1\right) M_{y}+l}(x, y)=j_{y j}^{r s}(x, y) \hat{\mathbf{y}}=-\frac{\mathrm{j} k_{0}}{2 s a_{j}} \\
& \times \frac{T_{r-1}\left(\frac{2(x-a / 2)}{a_{j}}\right)}{\sqrt{1-\left(\frac{2(x-a / 2)}{a_{j}}\right)^{2}}} U_{s-1}\left(\frac{2(y-b / 2)}{b_{j}}\right) \\
& \times \sqrt{1-\left(\frac{2(y-b / 2)}{b_{j}}\right)^{2}} \hat{\mathbf{y}} \\
& \left(j=1,2,3 ; r=1, \ldots, M_{x}\right. \text {; } \\
& \left.s=1, \ldots, M_{y}-1 ; l=1, \ldots, M_{x}\left(M_{y}-1\right)\right)
\end{aligned}
$$

where $T_{r-1}(\cdot)\left(T_{s-1}(\cdot)\right)$ and $U_{r-1}(\cdot)\left(U_{s-1}(\cdot)\right)$ are Chebyshev polynomials of first and second kind respectively, and where the total number of BFs per rectangular patch is $N_{b}=2 M_{x} M_{y}-$ $M_{x}-M_{y}$.

When the BFs of (30) and (31) are introduced in (14) and (15), and the results are subsequently introduced in (10) and (11), all the resulting integrals can be written as linear combinations of integrals of the type

$$
A_{p q, r s}^{i}=\int_{-a_{i}}^{a_{i}} \int_{-b_{i}}^{b_{i}} t_{p r}\left(x, a_{i}\right) t_{q s}\left(y, b_{i}\right) G_{0 i}^{\mathrm{pp}}(x, y) d x d y
$$

where the functions $t_{p r}\left(x, a_{i}\right)$ and $t_{q s}\left(y, b_{i}\right)$ were defined in [28, eq. (45)], and can be expressed in terms of the following set of integrals:

$$
t_{k l}(z, w)=\frac{\pi}{4 w} \mathrm{j}^{k-l} \int_{-\infty}^{+\infty} J_{k-1}(u) J_{l-1}(u) \mathrm{e}^{-\mathrm{j} \frac{2 z u}{w}} d u .
$$


In (33), $J_{k-1}(\cdot)\left(J_{l-1}(\cdot)\right)$ are Bessel functions of first kind and order $k-1(l-1)$. The products $t_{p r}\left(x, a_{i}\right) t_{q s}\left(y, b_{i}\right)$ of (32) are basically the cross-correlations of (14) and (15) for the particular $\mathrm{BF}$ of (30) and (31), and their representation in terms of (33) is just the spectral domain version of these crosscorrelations. The integrals of (33) can be obtained in closed form by means of the recurrent relations [28, eqs. (46)-(53)]. In particular, these integrals can all be written as linear combinations of complete elliptic integrals of the first and second kind. The functions $t_{k l}(z, w)$ of (33) turn out to have logarithmic singularities as $z \rightarrow 0$ when $k+l$ is even, and first derivative with logarithmic singularities as $z \rightarrow 0$ when $k+l$ is odd. This means the integrands of the 2-D integrals of (32) present logarithmic singularities or derivatives with logarithmic singularities when either $x=0$ or $y=0$, which may have a deleterious effect in the numerical computation of these integrals. These singularities must be located and carefully handled. The specific behavior of $t_{k l}(z, w)$ as $z \rightarrow 0$ is given by the expressions

$$
\begin{aligned}
\left.t_{k l}(z, w)\right|_{z \rightarrow 0(k+l \text { even })} & \left.\approx t_{k l}^{\text {sing }}(z, w)\right|_{k+l \text { even }} \\
& =-\frac{1}{2 w} \ln |z|+p_{k l} \\
\left.t_{k l}(z, w)\right|_{z \rightarrow 0(k+l \text { odd })} & \left.\approx t_{k l}^{\text {sing }}(z, w)\right|_{k+l \text { odd }} \\
& =\frac{q_{k l}}{4 w^{2}} z \ln |z|-\frac{r_{k l}}{4 w^{2}} z
\end{aligned}
$$

where $p_{k l}, q_{k l}$, and $r_{k l}(k, l=1, \ldots)$ are constant quantities that can be computed by means of recurrent relations that are provided in Appendix A.

The MPGF $G_{0 i}^{\mathrm{pp}}(x, y)$ of (32) are also singular when $x=$ $y=0$ (i.e., when $\rho=0$ ). According to (22), the singular behavior of $G_{0 i}^{\mathrm{pp}}(x, y)$ around $\rho=0$ can be written as

$$
\left.G_{0 i}^{\mathrm{pp}}(x, y)\right|_{\rho \approx 0} \approx G_{0 i}^{\mathrm{sing}}(x, y)=\frac{e_{i 0}}{2 \pi \rho}+K_{i}
$$

where the constant $K_{i}$ can be easily computed by means of (24) and (25).

If the integrals of (32) are computed by means of standard Gauss-Legendre quadratures, the singularities of (34), (35), and (36) will have a deleterious effect in the numerical integration process. In order to mitigate the effect of the singularities, we have split these integrals into two parts as follows:

$$
A_{p q, r s}^{i}=A_{p q, r s}^{i, \text { num }}+A_{p q, r s}^{i, \text { sing }}
$$

where

$$
\begin{aligned}
A_{p q, r s}^{i, \mathrm{num}}= & \int_{-a_{i}}^{a_{i}} \int_{-b_{i}}^{b_{i}}\left[t_{p r}\left(x, a_{i}\right) t_{q s}\left(y, b_{i}\right) G_{0 i}^{\mathrm{pp}}(x, y)\right. \\
& \left.-t_{p r}^{\mathrm{sing}}\left(x, a_{i}\right) t_{q s}^{\mathrm{sing}}\left(y, b_{i}\right) G_{0 i}^{\mathrm{sing}}(x, y)\right] d x d y \\
= & \int_{-a_{i}}^{a_{i}} \int_{-b_{i}}^{b_{i}} w_{p q, r s}^{i, \mathrm{num}}(x, y) d x d y
\end{aligned}
$$

and where

$$
A_{p q, r s}^{i, \text { sing }}=\int_{-a_{i}}^{a_{i}} \int_{-b_{i}}^{b_{i}} t_{p r}^{\text {sing }}\left(x, a_{i}\right) t_{q s}^{\text {sing }}\left(y, b_{i}\right) G_{0 i}^{\text {sing }}(x, y) d x d y \text {. }
$$

In this paper, the integrals $A_{p q, r s}^{i, n u m}$ are numerically computed. Although the singularity introduced by the three factors $t_{p r}\left(x, a_{i}\right), t_{q s}\left(y, b_{i}\right)$, and $G_{0 i}^{\mathrm{pp}}(x, y)$ at $x=y=0$ has been removed in $w_{p q, r s}^{i, n u m}(x, y)$, the logarithmic singularities of $t_{p r}\left(x, a_{i}\right)$ at $x=0$ when $y \neq 0$ and those of $t_{q s}\left(y, b_{i}\right)$ at $y=0$ when $x \neq 0$ are still present in $w_{p q, r s}^{i, \text { num }}(x, y)$. So, before proceeding with the numerical integration of (38), the integrals $A_{p q, r s}^{i, \text { num }}$ are rearranged in the following way:

$$
\begin{aligned}
& A_{p q, r s}^{i, \text { num }}=a_{i} b_{i} \int_{0}^{1} \int_{0}^{1}\left[w_{p q, r s}^{i, \text { num }}\left(x=a_{i} u, y=b_{i} v\right)\right. \\
& +w_{p q, r s}^{i, \text { num }}\left(x=-a_{i} u, y=b_{i} v\right)+w_{p q, r s}^{i, n u m}\left(x=a_{i} u, y=-b_{i} v\right) \\
& \left.+w_{p q, r s}^{i, \text { num }}\left(x=-a_{i} u, y=-b_{i} v\right)\right] d u d v
\end{aligned}
$$

The integrals of (40) still have logarithmic singularities for $u=0$ when $v \neq 0$, and for $v=0$ when $v \neq 0$. However, these singularities can be adequately handled by means of MRW quadrature rules [29] since these quadrature rules are specifically designed for integrals of functions with logarithmic singularities in the lower limit of the integration interval. In fact, the MRW quadrature rules have been successfully used in [30] for the determination of integrals with logarithmic singularities that arise in the application of the MoM to free space problems with subsectional triangular BF. Bearing in mind the capabilities of MRW quadratures to handle logarithmic singularities, the integrals of (40) have been computed by means of iterated MRW quadratures of order $N_{\text {MRW }}$ [which implies that the determination of the integrals has required $4 N_{\mathrm{MRW}}^{2}$ evaluations of the function $w_{p q, r s}^{i, n_{m}}(x, y)$ in accordance with (40)].

The integrals $A_{p q, r s}^{i, \text { sing }}$ of (39) turn out to be zero when $p+r$ and/or $q+s$ are odd numbers owing to the odd symmetry of the integrand with respect to $x$ and/or $y$ in those cases. However, the integrals $A_{p q, r s}^{i, \text { sing }}$ are not zero when both $p+r$ and/or $q+s$ are even numbers. For that particular case, we have developed a very efficient way to compute $A_{p q, r s}^{i, \operatorname{sing}}$. The details of these calculations are provided in Appendix B.

\section{Numerical RESUlts and VAlidations}

The multilayered substrate used in the results presented in this section is that used for the design of the reflectarray antenna of [5]. This is a nine-layer substrate $\left[N_{l}=9\right.$ in Fig. 1(a)] for which $d_{1}=d_{4}=d_{7}=2 \mathrm{~mm}, d_{2}=d_{5}=$ $d_{8}=0.085 \mathrm{~mm}, d_{3}=d_{6}=d_{9}=0.18 \mathrm{~mm}, \varepsilon_{r, 1}=\varepsilon_{r, 4}=\varepsilon_{r, 7}$ $=1.1, \varepsilon_{r, 2}=\varepsilon_{r, 5}=\varepsilon_{r, 8}=3.043, \varepsilon_{r, 3}=\varepsilon_{r, 6}=\varepsilon_{r, 9}=2.67$, $\tan \delta_{1}=\tan \delta_{4}=\tan \delta_{7}=0.002, \quad \tan \delta_{2}=\tan \delta_{5}=\tan \delta_{8}$ $=0.0036$, and $\tan \delta_{3}=\tan \delta_{6}=\tan \delta_{9}=0.0092$. A ground plane is located at the lower end of the substrate as usual in reflectarray applications. The patches used in the reflectarray antenna of [5] are placed at the interfaces that are two layers, five layers, and eight layers above the ground plane [i.e., $P_{1}=2, P_{2}=5, P_{3}=8$ in Fig. 1(a)], which means that very thin layers (around $0.1 \mathrm{~mm}$ thick) are located next to the metallizations. Numerical simulations have shown that for this particular multilayered substrate, when five exponential terms are retained in (16) $\left(N_{D}=5\right)$, this equation provides an accuracy of roughly five significant figures when $5 k_{0} \leq k_{\rho} \leq 10 k_{0}$, 


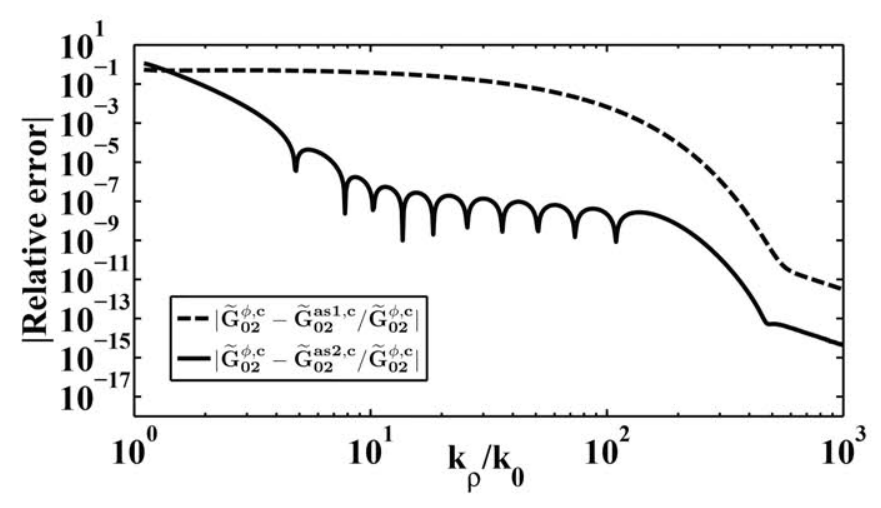

Fig. 2. Magnitude of the relative errors between the spectral domain multilayered Green's functions for the scalar potential and the asymptotic approximations of (21) (dashed line) and (28) (solid line). Parameters: $z=z^{\prime}=-h_{P_{2}}=$ $-2.445 \mathrm{~mm} ; f=12.1 \mathrm{GHz} ; N_{D}=5$.

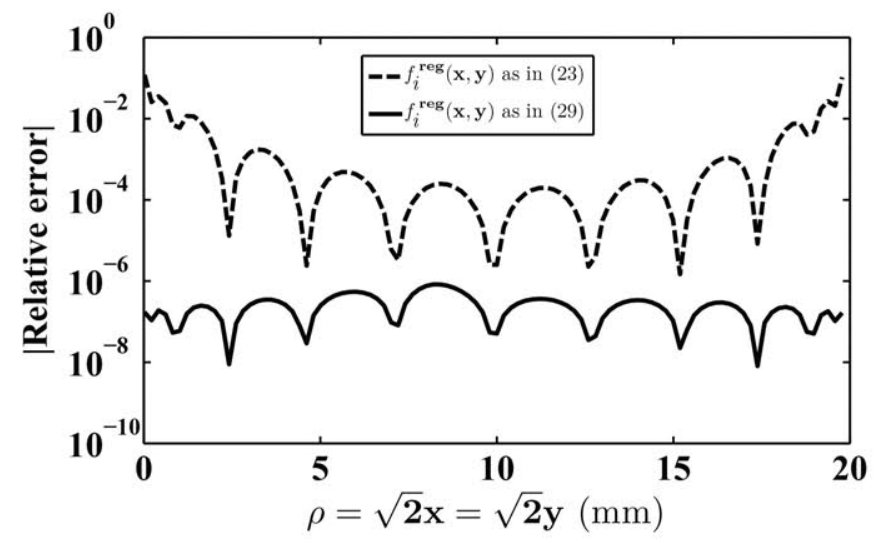

Fig. 3. Magnitude of the relative errors between the spatial domain MPGF for the scalar potential $G^{\phi, p p}$ and the interpolation obtained from (24) and (25) when the regularization functions $f_{i}^{\text {reg }}(x, y)$ are given either by (23) (dashed line), or by (29) (solid line). The MPGF values are sampled along the diagonal of the unit cell. Parameters: $z=z^{\prime}=-h_{P_{2}}=-2.445 \mathrm{~mm}$; $f=12.1 \mathrm{GHz} ; a=b=14 \mathrm{~mm} ; \theta_{\mathrm{i}}=\varphi_{\mathrm{i}}=30^{\circ} ; N_{D}=5 ; N_{c}=10$.

and a larger accuracy of roughly seven significant figures when $k_{\rho} \geq 10 k_{0}$.

In Fig. 2, the relative errors among the spectral Green's function $\widetilde{G}^{\phi, c}$ and the two asymptotic approximations of (21) and (28) versus $k_{\rho} / k_{0}$ are plotted. As expected, the larger the number of terms included in the asymptotic approximations, the larger the interval of $k_{\rho} / k_{0}$ accurately covered by these approximations. While the relative error between $\widetilde{G}^{\phi, c}$ and (21) is below $0.01 \%$ when $k_{\rho} \gtrsim 200 k_{0}$, the relative error between $\widetilde{G}^{\phi, \mathrm{c}}$ and (28) is below $0.01 \%$ when $k_{\rho} \gtrsim 5 k_{0}$. This indicates that in the case of multilayered substrates containing thin layers, whereas (21) is a poor asymptotic approximation of $\widetilde{G}^{\phi, c},(28)$ provides a sufficiently accurate approximation of $\widetilde{G}^{\phi, c}$ in a wide range of values of $k_{\rho} / k_{0}$ since it accounts for the effect of the quasidynamic images trough the thin layers in the spectral domain as commented in Section III.

Fig. 3 shows results for the interpolation of the regularized MPGF of (24) in terms of Chebyshev polynomials [see (25)] for the two different regularization functions of (23) and (29). As expected, the larger the number of terms included in the

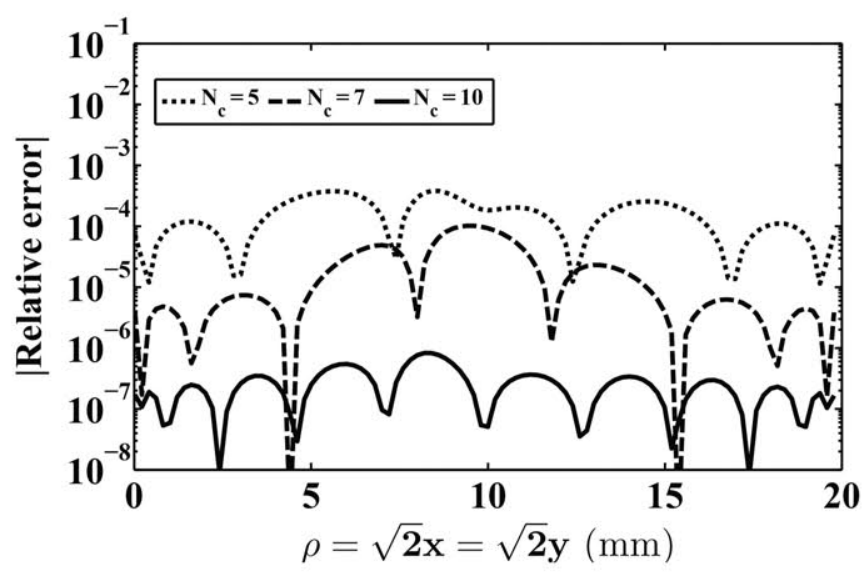

Fig. 4. Magnitude of the relative error arising from the interpolation of the MPGF $G^{\phi, p p}$ for different values of the number of Chebyshev polynomials used in each direction $N_{c}$. The interpolation is carried out by means of (24), (25), and (29). The MPFG values are sampled along the diagonal of the unit cell. Parameters: $z=z^{\prime}=-h_{P_{2}}=-2.445 \mathrm{~mm} ; f=12.1 \mathrm{GHz} ; a=b=$ $14 \mathrm{~mm} ; \theta_{\mathrm{i}}=\varphi_{\mathrm{i}}=30^{\circ} ; N_{D}=5$.

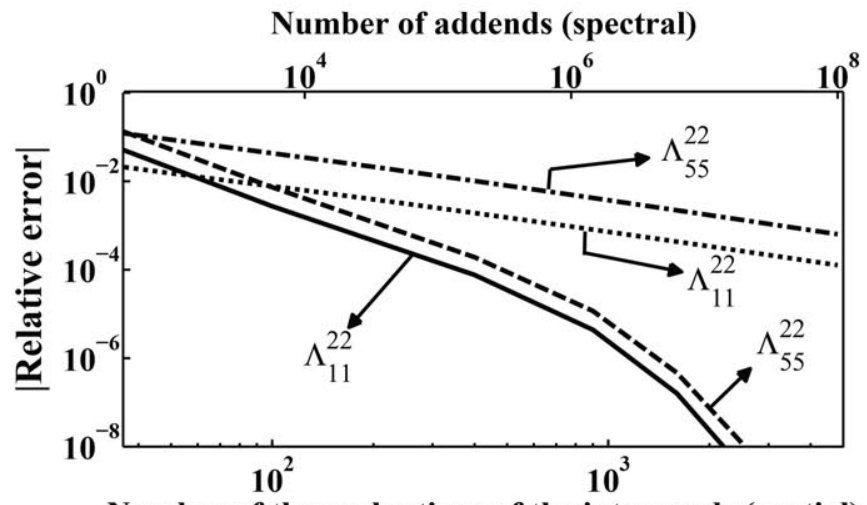

Number of the evaluations of the integrands (spatial)

Fig. 5. Magnitude of the relative error in the numerical computation of MoM matrix entries $\Lambda_{11}^{22}$ and $\Lambda_{55}^{22}$. The solid and dashed lines are obtained in the spatial domain by means of (9)-(11). The dotted and dashed-dotted lines are obtained by means of (7). In the spatial domain case, the relative error is plotted as a function of the number of the evaluations of the integrands in (10) and (11). In the spectral domain case, the relative error is plotted as a function of the number of addends retained in the series of (7). Parameters: $z=z^{\prime}=-h_{P_{2}}=$ $-2.445 \mathrm{~mm} ; f=12.1 \mathrm{GHz} ; a=b=14 \mathrm{~mm} ; \theta_{\mathrm{i}}=\varphi_{\mathrm{i}}=30^{\circ} ; a_{2}=b_{2}=$ $9.63 \mathrm{~mm} ; M_{x}=M_{y}=3$.

regularization functions of (24), the more accurate the representation of $G^{\phi, p p}$ around the source points, and therefore, the more accurate the interpolation of (25) for a given number of Chebyshev polynomials $\left(N_{c}=10\right.$ in this case). Note that the interpolation errors obtained with (23) reach $10 \%$ in the proximity of the sources for the substrate of [5] (which is not admissible) since (23) does not include the effect of the quasidynamic images through the thin layers. However, the interpolation errors obtained with (29) are always below $0.0001 \%$ in the whole range of values of $\rho$. In Fig. 4, the relative errors arising from the Chebyshev polynomial interpolation of (25) are plotted versus $N_{c}$ when the regularization function of (29) is used in (24). Note that the relative errors quickly 
decrease as the degrees of the polynomials increase. This indicates that the regularized MPGF are smooth functions, even in the neighborhood of the source points. Note that a value of $N_{c}=7$ suffices to obtain the interpolated MPGF with four significant figures. This means that only 64 samples of the MPGF are required to generate this interpolation, which is substantially less than the number of samples required in [25] and [26] to obtain the interpolated periodic Green's functions with the same accuracy of four significant figures.

In Fig. 5, the standard spectral domain MoM is compared with the spatial domain MoM formulated in this paper regarding the computation of the MoM entries $\Lambda_{11}^{22}$ and $\Lambda_{55}^{22}$ of (7) and (9). While the computation of these MoM entries with an accuracy of three significant figures in the spectral domain requires the summation of a few million terms in (7), the evaluation of the MoM entries with the same accuracy in the spatial domain only requires the computation of a few hundred integrands in the integrals of (10) and (11). Fig. 5 also shows that the slope of the convergence ratio of the MoM entries versus the number of quadrature points used in (10) and (11) is substantially larger than that of the convergence ratio versus the number of addends in the summations of (7).

In order to validate our MoM codes for the analysis of the structure of Fig. 1(a), in Fig. 6(a) and (b), we compute the magnitude and phase of the scattering parameter $S_{x x}$ for the multilayered periodic structure with three stacked patches used in the design of the reflectarray of [5]. This scattering parameter $S_{x x}$ is one of the diagonal elements of the cartesian scattering matrix defined in [39, eq. (1)]. The phase of these diagonal elements turns out to be crucial in the design of a reflectarray antenna [1], [39]. The phase curve of Fig. 6(b) is the phase-shift introduced by the reflectarray cell as a function of the patch size, which shows a range of phase variation larger than $700^{\circ}$ as typically occurs for a cell made of three stacked patches (see [3, Fig. 2]). The magnitude curve of Fig. 6(a) is used to estimate the effect of dielectric losses in the designed antenna. Our results obtained with the spectral MoM and with the hybrid spectralspatial MoM are compared with the commercial software CST. Excellent agreement is observed in the comparisons carried out.

Fig. 7(a) and (b) shows the convergence of MoM with respect to the number of BFs used in the calculation of the phase of the scattering parameter $S_{x x}$ plotted in Fig. 6(b) for two different sets of dimensions. Whereas normal incidence was considered in Fig. 6(a) and (b), oblique incidence has been considered to obtain the results of Fig. 7(a) and (b). The set of dimensions used in Fig. 7(a) $\left(a_{1}=b_{1}=9.85 \mathrm{~mm}\right)$ is in between the two sets of dimensions for which the periodic structure is resonant [where the magnitude of $S_{x x}$ reaches a minimum in Fig. 6(a)], and the set of dimensions used in Fig. 7(b) $\left(a_{1}=\right.$ $b_{1}=10.7 \mathrm{~mm}$ ) is the second set of resonant dimensions in Fig. 6(a). In the case of Fig. 7(a), the angle $\angle S_{x x}$ converges within two significant figures when about $24 \mathrm{BFs}$ of the type shown in (30) and (31) are used per patch in the structure of Fig. 1(a), and within three significant figures when about 100 BFs are used per patch. However, in the case of Fig. 7(b), about $60 \mathrm{BFs}$ are required to reach convergence within two significant figures, and about $150 \mathrm{BFs}$ are required to ensure convergence

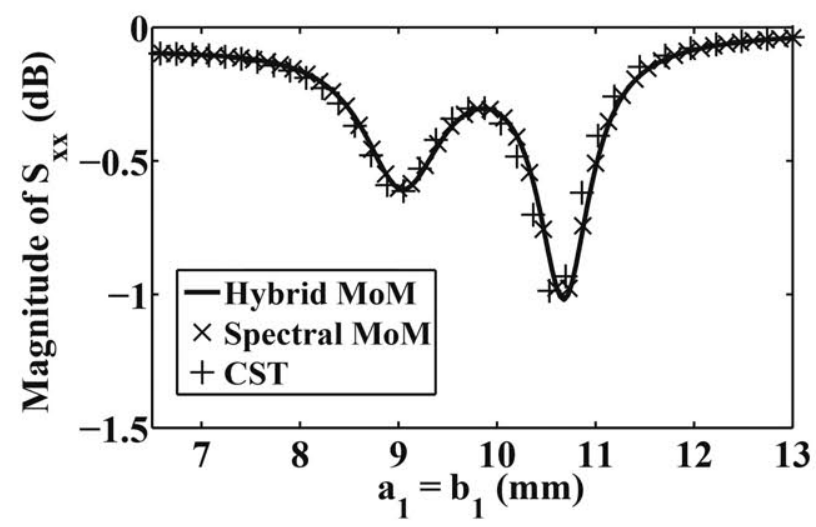

(a)

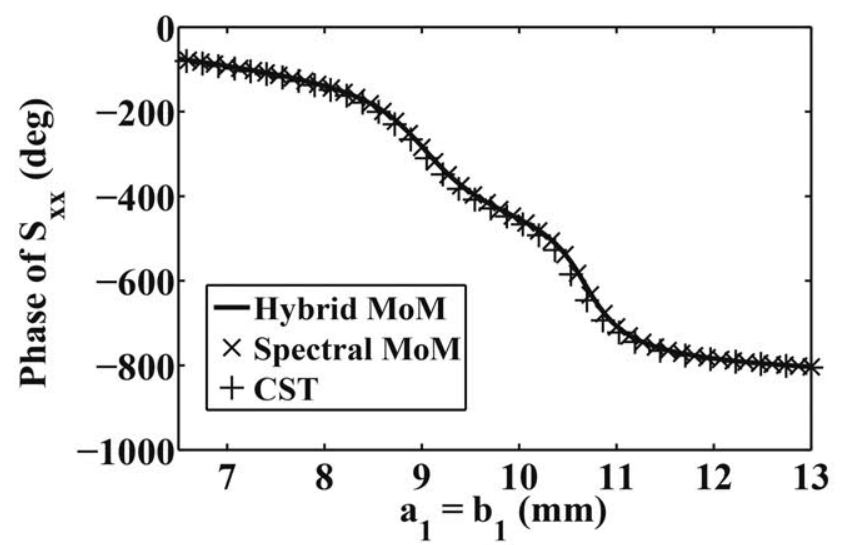

(b)

Fig. 6. Magnitude (a) and phase (b) of $S_{x x}$ for the reflectarray element made of three stacked patches that has been used in [5]. Our results obtained with both the spectral domain MoM $(\times)$ and the hybrid MoM of Sections II-IV (solid lines) are compared with those obtained with CST $(+)$. Parameters: $f=$ $12.1 \mathrm{GHz} ; a=b=14 \mathrm{~mm} ; \theta_{\mathrm{i}}=\varphi_{\mathrm{i}}=0^{\circ} ; a_{1}=b_{1} ; a_{2}=b_{2}=0.9 a_{1}$; $a_{3}=b_{3}=0.8 a_{1}$.

within three significant figures. In general, numerical simulations have shown that the convergence of MoM with respect to the number of BFs of (30) and (31) is very fast [similar to that of Fig. 7(a)], except for dimensions close to those of resonant structures where convergence becomes poorer [similar to that of Fig. 7(b)]. In Fig. 7(a) and (b), we also plot the ratio $\mathrm{T}_{\text {spe }} / \mathrm{T}_{\text {hyb }}$ between the CPU time required by the spectral domain MoM and that required by the hybrid spectral-spatial domain MoM of this paper to obtain $\angle S_{x x}$ with both two and three significant figures for a given number of BFs. Note that the hybrid MoM is between 40 and 80 times faster than the spectral MoM when an accuracy of two significant figures is required in the values of $\angle S_{x x}$, and between 2000 and 3000 times faster than the spectral MoM when an accuracy of three significant figures is required in the values of $\angle S_{x x}$.

In Fig. 8, we plot results for the radiation patterns of the reflectarray designed in [5] in the frequency band 11.45$11.7 \mathrm{GHz}$ for V-polarization, and in the frequency band 11.45 $12.75 \mathrm{GHz}$ for H-polarization. Starting from the exact dimensions obtained in [5] for the reflectarray elements (a total of 4068 elements), we have obtained the Cartesian scattering 


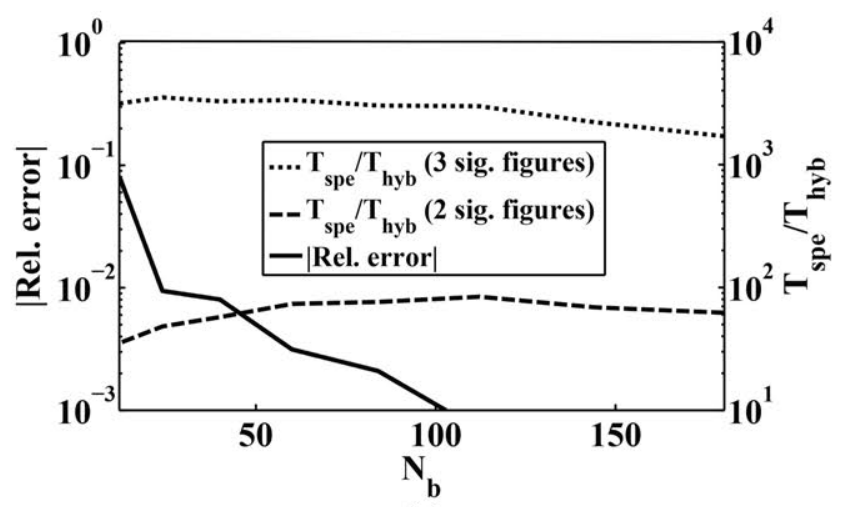

(a)

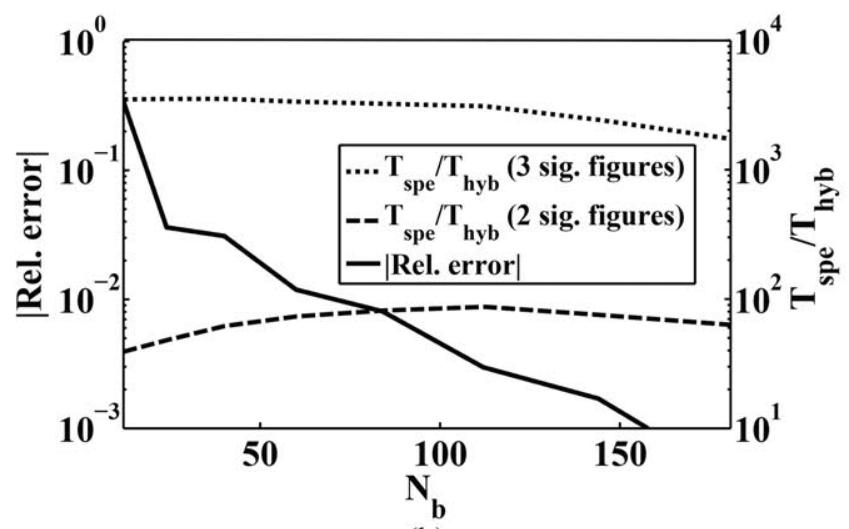

(b)

Fig. 7. Solid line stands for the magnitude of the relative error in the MoM determination of $\angle S_{x x}$ versus the number of BFs per patch $N_{b}$. The dashed line stands for the ratio $\mathrm{T}_{\mathrm{spe}} / \mathrm{T}_{\mathrm{hyb}}$ between the CPU time required by the spectral domain MoM to obtain $\angle S_{x x}$ with an accuracy of two significant figures and that required by the hybrid MoM of Sections II-IV. The dotted line stands for $\mathrm{T}_{\text {spe }} / \mathrm{T}_{\text {hyb }}$ when an accuracy of three significant figures is required in $\angle S_{x x}$. The results of (a) and (b) are for the nine-layer substrate used in [5]. Parameters: $f=12.1 \mathrm{GHz} ; a=b=14 \mathrm{~mm} ; \theta_{\mathrm{i}}=\varphi_{\mathrm{i}}=30^{\circ} ; a_{1}=b_{1}=9.85 \mathrm{~mm}$ in (a) and $a_{1}=b_{1}=10.7 \mathrm{~mm}$ in (b); $a_{2}=b_{2}=0.9 a_{1} ; a_{3}=b_{3}=0.8 a_{1}$.

matrix [39] for each of these elements under the local periodicity assumption, and from these scattering matrices, we have obtained the radiation patterns as explained in [1]. The computation of the scattering matrices has been carried out with both the spectral MoM and the hybrid MoM. A maximum relative error of $5 \%$ has been allowed in the computation of the elements of the scattering matrices since this suffices for a graphical representation such as that shown in Fig. 8. In particular, in the calculations we have retained 10000 addends in the summations of (7) for the spectral domain computation of $\Lambda_{k l}^{i i}$, and we have used $24 \mathrm{BFs}$ per patch, $N_{c}=5$ in the Chebyshev interpolations of (25), and $N_{\mathrm{MRW}}=5$ for the computation of the integrals of (40). Note that in Fig. 8, the radiation pattern results obtained with both the spectral MoM and the hybrid MoM match very well the measurements reported in [5]. The ratio $\mathrm{T}_{\text {spe }} / \mathrm{T}_{\text {hyb }}$ between the CPU time required by the spectral MoM and the CPU time required by the hybrid MoM for the analysis of the antenna of [5] has turned out to be around 10. This CPU time ratio is smaller

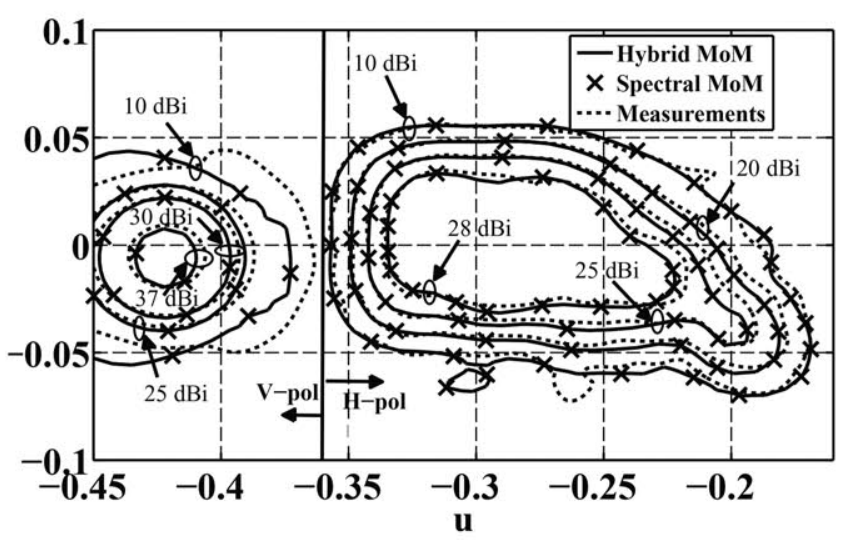

Fig. 8. Copolar gain patterns of the reflectarray designed in [5] for Vpolarization (left) at $11.575 \mathrm{GHz}$ and $\mathrm{H}$-polarization (right) at $12.1 \mathrm{GHz}$. This reflectarray has been analyzed with our codes, and the results obtained with both the spectral domain MoM $(x)$ and the hybrid MoM of Sections II to IV (solid lines) under the local periodicity assumption are compared with measurements [5] (dashed lines).

than those shown in Fig. 7(a) and (b), but this is because in Fig. 8, a larger error has been allowed in the computation of the cartesian scattering matrix and a smaller number of terms has been required in the summations of (7), which is beneficial for the spectral MoM. Besides analyzing the antenna of [5, Fig. 8], in Fig. 9, we plot the radiation patterns obtained for an independent design of this antenna carried out at only two frequencies, $11.575 \mathrm{GHz}$ for V-polarization and $12.1 \mathrm{GHz}$ for H-polarization. In this case, the dimensions of the reflectarray elements have been adjusted with both the spectral MoM code and the hybrid MoM code to obtain the required reflection phases that lead to the radiation patterns shown in the templates of Fig. 9. In particular, in the current design, we have used the reflection phases shown in [5, Fig. 6] at $11.575 \mathrm{GHz}$ for Vpolarization, and in [5, Fig. 3] at $12.1 \mathrm{GHz}$ for H-polarization. As in the case of the analysis carried out in Fig. 8, a maximum relative error of $5 \%$ has been allowed in the computation of the elements of the scattering matrices that have been used for the antenna design of Fig. 9. In spite of this, the ratio $\mathrm{T}_{\text {spe }} / \mathrm{T}_{\text {hyb }}$ between the CPU times required by the two codes to perform the antenna design is around 30 , which is larger than that obtained for the analysis of Fig. 8. The explanation for this is that for every reflectarray element and every direction of incidence, whereas the analysis of the antenna only requires the analysis of one periodic structure, the design requires the analysis of several periodic structures since the dimensions have to be adjusted several times until the correct reflection phase is achieved. While the dimensions are being adjusted, the MPGF do not vary and their interpolation has to be performed only once, which leads to important CPU time savings. So, in the case of the analysis of a reflectarray antenna, the number of MPGF interpolations equals the number of periodic structures that have to be studied. However, in the case of the design of a reflectarray antenna, the number of periodic structures to be studied is several times larger than the number of MPGF interpolations. 


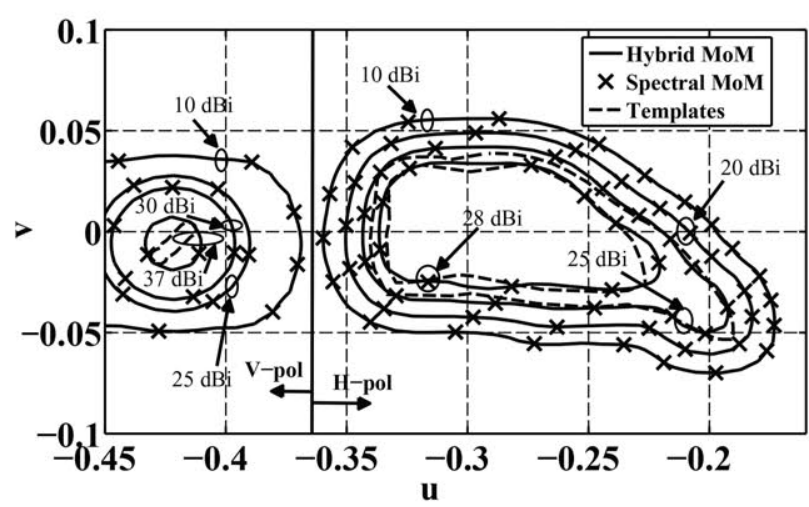

Fig. 9. Copolar gain patterns of the reflectarray designed in this paper at two frequencies: $11.575 \mathrm{GHz}$ for V-polarization (left) and $12.1 \mathrm{GHz}$ for $\mathrm{H}$ polarization (right). Our results obtained with both the spectral domain MoM $(x)$ and the hybrid MoM of Sections II-IV (solid lines) under the local periodicity assumption are compared with the templates used for the design (dashed lines). The multilayered substrate employed for the design is the nine-layer substrate of [5].

\section{CONCLUSION}

In this paper, a novel hybrid spectral-spatial MoM approach is presented for the efficient analysis of periodic arrays of rectangular stacked patches in multilayered substrates. This approach is expected to save CPU time in the design of large reflectarray antennas using those stacked patches. In the approach, the MoM matrix entries involving BF from two different metallization levels are computed in the spectral domain, and the MoM matrix entries involving BF from the same metallization level are computed in the spatial domain. In order to ensure an efficient computation of the spatial MoM matrix entries, the spatial domain MPGF are interpolated in terms of a small number of Chebyshev polynomials after analytically extracting the behavior of the MPFG around the source points. Also, the singularities of the integrals leading to the spatial MoM entries are either analytically extracted or numerically handled by means of MRW quadrature rules. Numerical simulations have shown that the CPU time required by the hybrid MoM in the analysis of one periodic structure made of stacked patches is around 60 times smaller than that required by the standard spectral MoM for an accuracy of two significant figures. Also, the hybrid MoM has been applied to the design of a contoured beam reflectarray antenna containing 4086 elements, and the CPU time required by the hybrid MoM introduced in this paper has been found to be around 30 times smaller than that required by the standard spectral MoM.

\section{APPENDIX A}

The coefficients $p_{k l}(k+l$ even) defined in (34) can be expressed in terms of new coefficients $u_{k l}(k+l$ even $)$ as follows:
The coefficients $u_{k k}$, which are particular cases of the coefficients $u_{k l}$ of (41), are given by the recurrent relation

$$
\begin{aligned}
u_{k k}= & \frac{1}{2 k-3}\left\{(4 k-8) u_{k-1, k-1}\right. \\
& \left.-(2 k-5) u_{k-2, k-2}\right\} \quad(k \geq 3)
\end{aligned}
$$

which is initialized by

$$
\begin{aligned}
& u_{11}=0 \\
& u_{22}=-2 .
\end{aligned}
$$

Once the coefficients $u_{k k}$ have been obtained $(k=1, \ldots)$, the coefficients $u_{k l}(k<l ; k+l$ even) can be obtained by means of the coupled recurrent relations

$$
\begin{aligned}
& u_{1 l}=2 u_{2, l-1}-u_{1, l-2} \quad(l \geq 3) \\
& u_{k l}=u_{k-1, l-1}+u_{k+1, l-1}-u_{k, l-2} \quad(l-1>k \geq 2) .
\end{aligned}
$$

Finally, the coefficients $u_{k l}(k>l ; k+l$ even) can be obtained from the coefficients $u_{k l}$ ( $k<l ; k+l$ even) derived from (45) and (46) by invoking the relation

$$
u_{k l}=u_{l k}
$$

Once the coefficients $u_{k l}$ have been obtained, the coefficients $q_{k, k+1}$ and $r_{k, k+1}$, which are particular cases of the coefficients $q_{k l}$ and $r_{k l}(k+l$ odd) of (35), can be computed by means of the recurrent relations

$$
\begin{aligned}
& q_{k, k+1}=4+q_{k-1, k} \quad(k \geq 2) \\
& r_{k, k+1}=4 \ln (4 w)+4 u_{k k}+r_{k-1, k} \quad(k \geq 2)
\end{aligned}
$$

which are initialized by

$$
\begin{aligned}
& q_{12}=2 \\
& r_{12}=2 \ln (4 w) .
\end{aligned}
$$

Once the coefficients $u_{k l}, q_{k, k+1}$ and $r_{k, k+1}(k, l=1, \ldots)$ have all been computed, the coefficients $q_{k l}$ and $r_{k l}(k<l ; k+$ $l$ odd) of (35) can be obtained by means of the coupled recurrent relations

$$
\begin{aligned}
q_{1 l}= & 8+2 q_{2, l-1}-q_{1, l-2} \quad(l \geq 4) \\
q_{k l}= & 8+q_{k-1, l-1}+q_{k+1, l-1}-q_{k, l-2} \quad(l-2>k \geq 2) \\
r_{1 l}= & 8 \ln (4 w)+8 u_{1, l-1}+2 r_{2, l-1}-r_{1, l-2} \quad(l \geq 4) \\
r_{k l}= & 8 \ln (4 w)+8 u_{k, l-1}+r_{k-1, l-1} \\
& +r_{k+1, l-1}-r_{k, l-2} \quad(l-2>k \geq 2) .
\end{aligned}
$$

Finally, the coefficients $q_{k l}$ and $r_{k l}(k>l ; k+l$ odd) of (35) can be obtained from the coefficients $q_{k l}$ and $r_{k l}(k<l ; k+l$ odd) derived from (48) to (55) by invoking the relations

$$
\begin{aligned}
& q_{k l}=-q_{l k} \\
& r_{k l}=-r_{l k} .
\end{aligned}
$$




\section{APPENDIX B}

The integrals $A_{p q, r s}^{i, s i n g}$ defined in (39) are different from zero in the case where both $p+r$ and $q+s$ are even. In this particular case, the integrals $A_{p q, r s}^{i, \operatorname{sing}}$ can be evaluated in an efficient way as shown in this Appendix. According to (34), (36), and (39), if

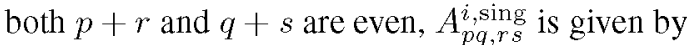

$$
\begin{aligned}
& A_{p q, r s}^{i, \text { sing }}=\int_{-a_{i}}^{a_{i}} \int_{-b_{i}}^{b_{i}}\left(-\frac{1}{2 w} \ln |x|+p_{p r}\right) \\
& \quad \times\left(-\frac{1}{2 w} \ln |y|+p_{q s}\right)\left(\frac{e_{i 0}}{2 \pi \sqrt{x^{2}+y^{2}}}+K_{i}\right) d x d y \\
& =\frac{e_{i 0}}{2 \pi w^{2}} \Omega_{i 1}-\frac{e_{i 0}}{\pi w}\left(p_{q s} \Omega_{i 2}+p_{p r} \Omega_{i 3}\right)+\frac{2 e_{i 0} p_{p r} p_{q s}}{\pi} \Omega_{i 4} \\
& \quad+\frac{K_{i} a_{i} b_{i}}{w^{2}}\left(\ln a_{i}-1\right)\left(\ln b_{i}-1\right)-\frac{2 K_{i} a_{i} b_{i}}{w}\left[p_{q s}\left(\ln a_{i}-1\right)\right. \\
& \left.\quad+p_{p r}\left(\ln b_{i}-1\right)\right]+4 K_{i} p_{q s} p_{p r} a_{i} b_{i}
\end{aligned}
$$

where

$$
\begin{aligned}
\Omega_{i 1} & =\int_{0}^{a_{i}} \int_{0}^{b_{i}} \frac{\ln |x| \ln |y|}{\sqrt{x^{2}+y^{2}}} d x d y \\
\Omega_{i 2} & =\int_{0}^{a_{i}} \int_{0}^{b_{i}} \frac{\ln |x|}{\sqrt{x^{2}+y^{2}}} d x d y \\
\Omega_{i 3} & =\int_{0}^{a_{i}} \int_{0}^{b_{i}} \frac{\ln |y|}{\sqrt{x^{2}+y^{2}}} d x d y \\
\Omega_{i 4} & =\int_{0}^{a_{i}} \int_{0}^{b_{i}} \frac{d x d y}{\sqrt{x^{2}+y^{2}}} \\
& =a_{i} \ln \left[\frac{\sqrt{a_{i}^{2}+b_{i}^{2}}+b_{i}}{a_{i}}\right]-b_{i} \ln \left[\frac{\sqrt{a_{i}^{2}+b_{i}^{2}}-a_{i}}{b_{i}}\right]
\end{aligned}
$$

The integrals (59)-(61) cannot be obtained in closed form. However, if polar variables are introduced $(x=\rho \cos \varphi$ and $y=$ $\rho \sin \varphi$ ), the integration with respect to $\rho$ can be carried out in closed form, and after some manipulations, $\Omega_{i 1}, \Omega_{i 2}$, and $\Omega_{i 3}$ can be rewritten as

$$
\begin{aligned}
\Omega_{i 1}= & a_{i}\left\{\left[\left(\ln a_{i}\right)^{2}-2 \ln a_{i}+2\right] \ln \left[\frac{\sqrt{a_{i}^{2}+b_{i}^{2}}+b_{i}}{a_{i}}\right]\right. \\
& \left.+\left(\ln a_{i}-1\right)\left(\Gamma_{i 1}-\Gamma_{i 2}\right)\right\}+b_{i}\left\{-\left[\left(\ln b_{i}\right)^{2}-2 \ln b_{i}+2\right]\right. \\
& \left.\times \ln \left[\frac{\sqrt{a_{i}^{2}+b_{i}^{2}}-a_{i}}{b_{i}}\right]+\left(\ln b_{i}-1\right)\left(\Gamma_{i 3}-\Gamma_{i 4}\right)\right\}
\end{aligned}
$$

$$
\begin{aligned}
\Omega_{i 2}= & a_{i}\left(\ln a_{i}-1\right) \ln \left[\frac{\sqrt{a_{i}^{2}+b_{i}^{2}}+b_{i}}{a_{i}}\right]+b_{i}\left\{-\left(\ln b_{i}-1\right)\right. \\
& \left.\times \ln \left[\frac{\sqrt{a_{i}^{2}+b_{i}^{2}}-a_{i}}{b_{i}}\right]+\left(\Gamma_{i 3}-\Gamma_{i 4}\right)\right\}
\end{aligned}
$$

$$
\begin{aligned}
\Omega_{i 3}= & a_{i}\left\{\left(\ln a_{i}-1\right) \ln \left[\frac{\sqrt{a_{i}^{2}+b_{i}^{2}}+b_{i}}{a_{i}}\right]+\left(\Gamma_{i 1}-\Gamma_{i 2}\right)\right\} \\
& -b_{i}\left(\ln b_{i}-1\right) \ln \left[\frac{\sqrt{a_{i}^{2}+b_{i}^{2}}-a_{i}}{b_{i}}\right]
\end{aligned}
$$

where $\Gamma_{i j}(j=1,2,3,4)$ are 1-D integrals that cannot be obtained in closed form and are given by

$$
\begin{aligned}
& \Gamma_{i 1}=\int_{0}^{\varphi_{i}} \frac{\ln (\sin \varphi)}{\cos \varphi} d \varphi=\int_{0}^{\sin \varphi_{i}} \frac{\ln t}{1-t^{2}} d t \\
& \Gamma_{i 2}=\int_{0}^{\varphi_{i}} \frac{\ln (\cos \varphi)}{\cos \varphi} d \varphi=\int_{\cos \varphi_{i}}^{1} \frac{\ln t}{t \sqrt{1-t^{2}}} d t \\
& \Gamma_{i 3}=\int_{\varphi_{i}}^{\pi / 2} \frac{\ln (\cos \varphi)}{\sin \varphi} d \varphi=\int_{0}^{\cos \varphi_{i}} \frac{\ln t}{1-t^{2}} d t \\
& \Gamma_{i 2}=\int_{\varphi_{i}}^{\pi / 2} \frac{\ln (\sin \varphi)}{\sin \varphi} d \varphi=\int_{\sin \varphi_{i}}^{1} \frac{\ln t}{t \sqrt{1-t^{2}}} d t
\end{aligned}
$$

where $\varphi_{i}=\tan ^{-1}\left(b_{i} / a_{i}\right)$.

While the integrands of $\Gamma_{i 2}$ and $\Gamma_{i 4}$ do not present singularities in the integration interval, the integrands of $\Gamma_{i 1}$ and $\Gamma_{i 3}$ show a logarithmic singularity when $t=0$. Numerical simulations have shown that all these integrals can be computed within five significant figures with a 20-point MRW quadrature rule when $4^{\circ} \leq \varphi_{i} \leq 86^{\circ}$, which covers most practical dimensions of rectangular patches.

One good thing about the computation of $A_{p q, r s}^{i, \text { sing }}$ by means of (58) is that once the dimensions of the $i$ th patch $a_{i}$ and $b_{i}$ are fixed, the integrals $\Omega_{i j}(j=1,2,3)$ only have to be computed once [via (63)-(69)], no matter how many BFs have been employed to model the current density on that particular patch. This means that only four 1-D numerical integrals (that are computed to a high accuracy with only 20 quadrature points) are required to obtain all the integrals $A_{p q, r s}^{i, \text { sing involved }}$ in the determination of the $N_{b}^{2}$ Galerkin's matrix entries $\Lambda_{k l}^{i i}$ $\left(k, l=1, \ldots, N_{b}\right)$ of $(9)$. Therefore, the CPU time required for

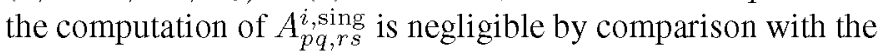
overall CPU time required for the computation of $\Lambda_{k l}^{i i}$.

\section{REFERENCES}

[1] J. Huang and J. A. Encinar, Reflectarray Antennas. Piscataway, NJ, USA: IEEE Press/Wiley, 2008.

[2] J. A. Encinar, "Design of two-layer printed reflectarrays using patches of variable size," IEEE Trans. Antennas Propag., vol. 49, no. 10, pp. 14031410 , Oct. 2001.

[3] J. A. Encinar and J. A. Zornoza, "Broadband design of three-layer printed reflectarrays," IEEE Trans. Antennas Propag., vol. 51, no. 7, pp. 16621664, Jul. 2003.

[4] J. A. Encinar and J. A. Zornoza, "Three-layer printed reflectarrays for contoured beam space applications," IEEE Trans. Antennas Propag., vol. 52, no. 5, pp. 1138-1148, May 2004.

[5] J. A. Encinar et al., "Dual-polarization dual-coverage reflectarray for space applications," IEEE Trans. Antennas Propag., vol. 54, no. 10, pp. 2828-2837, Oct. 2006.

[6] M. Arrebola, J. A. Encinar, and M. Barba, "Multifed printed reflectarray with three simultaneous shaped beams for LMDS central station antenna," IEEE Trans. Antennas Propag., vol. 56, no. 6, pp. 1518-1527, Jun. 2008. 
[7] J. A. Encinar, M. Arrebola, L. F. de la Fuente, and G. Tosso, "A transmitreceive reflectarray antenna for direct broadcast satellite applications," IEEE Trans. Antennas Propag., vol. 59, no. 9, pp. 3255-3264, Sep. 2011.

[8] D. M. Pozar and T. A. Metzler, "Analysis of a reflectarray antenna using microstrip patches of variable size," Electron. Lett., vol. 29, pp. 657-658, Apr. 1993.

[9] S. D. Targonski and D. M. Pozar, "Analysis and design of a microstrip reflectarray using patches of variable size," in Proc. IEEE Int. Symp. Antennas Propag., Seattle, WA, USA, Jun. 1994, pp. 1820-1823.

[10] D. M. Pozar, S. D. Targonski, and H. D. Syrigos, "Design of millimeter wave microstrip reflectarray," IEEE Trans. Antennas Propag., vol. 45 no. 2, pp. 287-296, Feb. 1997.

[11] M. Zhou et al., "Direct optimization of printed reflectarrays for contoured beam satellite antenna applications," IEEE Trans. Antennas Propag., vol. 61, no. 4, pp. 1995-2004, Apr. 2013.

[12] R. Mittra, C. H. Chan, and T. Cwik, "Techniques for analyzing frequency selective surfaces-A review," Proc. IEEE, vol. 76, no. 12, pp. 1593-1615, Dec. 1988.

[13] C. H. Chan, "A numerically efficient technique for the method of moments solution of electromagnetic problem associated with planar periodic structures," Microw. Opt. Technol. Lett., vol. 1, no. 10, pp. 372 374, Dec. 1988.

[14] C. H. Chan and R. Mittra, "On the analysis of frequency-selective surfaces using subdomain basis functions," IEEE Trans. Antennas Propag., vol. 38, no. 1, pp. 40-50, Jan. 1990.

[15] S. Hashemi-Yeganeh, "On the summation of double infinite series field computation inside rectangular cavities," IEEE Trans. Microw. Theory Tech., vol. 43, no. 3, pp. 641-646, Mar. 1995.

[16] R. R. Boix, M. Freire, and F. Medina, "New method for the efficient summation of double infinite series arising from the spectral domain analysis of frequency selective surfaces," IEEE Trans. Antennas Propag., vol. 52 , no. 4, pp. 1080-1094, Apr. 2004.

[17] W. C. Chew and Q. Liu, "Resonance frequency of a rectangular microstrip patch," IEEE Trans. Antennas Propag., vol. 36, no. 8, pp. 1045-1056, Aug. 1988.

[18] R. A. Kipp and C. H. Chan, "A numerically efficient technique for the method of moments solution for planar periodic structures in layered media," IEEE Trans. Microw. Theory Tech., vol. 42, no. 4, pp. 635-643, Apr. 1994.

[19] K. A. Michalski and J. R. Mosig, "Multilayered media Green's func tions in integral equation formulations," IEEE Trans. Antennas Propag., vol. 45, no. 3, pp. 508-519, Mar. 1997.

[20] A. L. Fructos, R. R. Boix, and F. Mesa, "Efficient computation of the off-diagonal elements of the vector-potential multilayered periodic dyadic Green's function," IEEE Trans. Antennas Propag., vol. 59, no. 7, pp. 2557-2564, Jul. 2011.

[21] M. A. Khayat and D. A. Wilton, "Numerical evaluation of singular and near-singular potential integrals," IEEE Trans. Antennas Propag., vol. 53 no. 10 , pp. $3180-3190$, Oct. 2005.

[22] A. G. Polimeridis, J. A. Tamayo, J. M. Rius, and J. R. Mosig, "Fast and accurate computation of Hypersingular integrals in Galerkin surface integral equation formulations via the direct evaluation method," IEEE Trans. Antennas Propag., vol. 59, no. 6, pp. 2329-2340, Jun. 2011.

[23] A. G. Polimeridis, F. Vipiana, J. R. Mosig, and D. R. Wilton, "DIRECTFN: Fully numerical algorithms for high precision computation of singular integrals in Galerkin SIE methods," IEEE Trans. Antennas Propag., vol. 61, no. 6, pp. 3112-3122, Jun. 2013.

[24] A. G. Polimeridis and J. R. Mosig, "Evaluation of weakly singular integrals via generalized cartesian product rules based on the double exponential formula," IEEE Trans. Antennas Propag., vol. 58, no. 6 , pp. 1980-1988, Jun. 2010.

[25] G. Valerio, P. Baccarelli, S. Paulotto, F. Frezza, and A. Galli, "Regularization of mixed-potential layered-media Green's functions for efficient interpolation procedures in planar periodic structures," IEEE Trans. Antennas Propag., vol. 57, no. 1, pp. 122-134, Jan. 2009.

[26] S. Li, D. Van Orden, and V. Lomakin, "Fast periodic interpolation method for periodic unit cell problems," IEEE Trans. Antennas Propag., vol. 58 no. 12 , pp. $4005-4014$, Dec. 2010.

[27] A. M. Lerer and A. G. Schuschinsky, "Full-wave analysis of threedimensional planar structures," IEEE Trans. Microw. Theory Tech., vol. 41, no. 11, pp. 2002-2015, Nov. 1993.

[28] R. Florencio, R. R. Boix, and J. A. Encinar, "Enhanced MoM analysis of the scattering by periodic strip gratings in multilayered substrates," IEEE Trans. Antennas Propag., vol. 61, no. 10, pp. 5088-5099, Oct. 2013.
[29] J. Ma, V. Rokhlin, and S. Wandzura, "Generalized gaussian quadrature rules for systems of arbitrary functions," SIAM J. Numer. Anal., vol. 33, no. 3, pp. 971-996, Jun. 1996.

[30] F. Vipiana, D. R. Wilton, and W. A. Johnson, "Advanced numerical schemes for the accurate evaluation of $4-\mathrm{D}$ reaction integrals in the method of moments," IEEE Trans. Antennas Propag., vol. 61, no. 11, pp. 5559-5566, Nov. 2013.

[31] F. Mesa, R. Marqués, and M. Horno, "A general algorithm for computing the bidimensional spectral Green's dyads in multilayered complex bianisotropic media: The equivalent boundary method," IEEE Trans. Microw. Theory Tech., vol. 39, no. 9, pp. 1640-1649, Sep. 1991.

[32] G. Valerio, P. Baccarelli, P. Burghignoli, and A. Galli, "Comparative analysis of acceleration techniques for 2-D and 3-D Green's functions in periodic structures along one and two directions," IEEE Trans. Antennas Propag., vol. 55, no. 6, pp. 1630-1643, Jun. 2007.

[33] M. I. Aksun, "A robust approach for the derivation of closed-form Green's functions," IEEE Trans. Microw. Theory Tech., vol. 44, no. 5, pp. 651-658, May 1996.

[34] M. J. Park and S. Nam, "Efficient calculation of the Green's function for multilayered planar periodic structures," IEEE Trans. Antennas Propag., vol. 46 , no. 10 , pp. $1582-1583$, Oct. 1998

[35] Y. Hua and T. K. Sarkar, "Generalized pencil-of-function method for extracting poles of an EM system from its transient response," IEEE Trans. Antennas Propag., vol. 37, no. 2, pp. 229-234, Feb. 1989

[36] K. E. Jordan, G. E. Richter, and P. Sheng, "An efficient numerical evaluation of the Green's function for the Helmholtz operator in periodic structures," J. Comput. Phys., vol. 63, pp. 222-235, 1986.

[37] L. S. Gradshteyn and L. M. Ryzhik, Table of Integrals, Series and Products, 6th ed. New York, NY, USA: Academic, 2000

[38] Y. L. Chow, J. J. Yang, D. G. Fang, and G. E. Howard, "A closed-form spatial Green's function for the thick microstrip substrate," IEEE Trans. Microw. Theory Tech., vol. 39, no. 3, pp. 588-592, Mar. 1991.

[39] R. Florencio, R. R. Boix, E. Carrasco, J. A. Encinar, and V. Losada, "Efficient numerical tool for the analysis and design of reflectarrays based on cells with three parallel dipoles," Microw. Opt. Technol. Lett., vol. 55, no. 6 , pp. 1212-1216, Jun. 2013. 